\title{
ROBUST MPC FOR ACTUATOR-FAULT TOLERANCE USING SET-BASED PASSIVE FAULT DETECTION AND ACTIVE FAULT ISOLATION
}

\author{
Feng XU $^{a, d}$, Vicenç PUIG ${ }^{a}$, CARLOS OCAMPO-MARTINEZ $^{a, *}$, SORIN OLARU $^{b}$, \\ SILVIU-IULIAN NICULESCU ${ }^{c}$
}

\author{
${ }^{a}$ Institut de Robòtica i Informàtica Industrial (CSIC-UPC) \\ Technical University of Catalonia (UPC), Llorens i Artigas, 4-6, 08028 Barcelona, Spain \\ e-mail $\{$ vpuig, cocampo\}@iri.upc.edu \\ ${ }^{b}$ Automatic Control Department \\ E3S (Supélec Systems Sciences), 3 rue Joliot-Curie, 91192 Gif sur Yvette, Paris, France
}

${ }^{c}$ Laboratory of Signals and Systems

CNRS—Centrale Supélec, 3 rue Joliot Curie, 91192 Gif sur Yvette, Paris, France

${ }^{d}$ Center of Intelligent Control and Telescience, Graduate School at Shenzhen

Tsinghua University, University Town, Nanshan, 518055 Shenzhen, PR China

\begin{abstract}
In this paper, a fault-tolerant control (FTC) scheme is proposed for actuator faults, which is built upon tube-based model predictive control (MPC) as well as set-based fault detection and isolation (FDI). In the class of MPC techniques, tubebased MPC can effectively deal with system constraints and uncertainties with relatively low computational complexity compared with other robust MPC techniques such as min-max MPC. Set-based FDI, generally considering the worst case of uncertainties, can robustly detect and isolate actuator faults. In the proposed FTC scheme, fault detection (FD) is passive by using invariant sets, while fault isolation (FI) is active by means of MPC and tubes. The active FI method proposed in this paper is implemented by making use of the constraint-handling ability of MPC to manipulate the bounds of inputs. After the system has been detected to become faulty, the input-constraint set of the MPC controller is adjusted to actively excite the system for achieving FI guarantees on-line, where an active FI-oriented input set is designed off-line. In this way, the system can be excited in order to obtain more extra system-operating information for FI than passive FI approaches. Overall, the objective of this paper is to propose an actuator MPC scheme with as little as possible of FI conservatism and computational complexity by combining tube-based MPC and set theory within the framework of MPC, respectively. Finally, a case study is used to show the effectiveness of the proposed FTC scheme.
\end{abstract}

Keywords: fault detection, fault isolation, set-theoretic method, fault-tolerant control, model predictive control.

\section{Introduction}

In general, all technical systems are prone to faults. In a controlled system, if the plant itself is more reliable than the sensors and actuators used, when the closed-loop performance deviates from its normal situation, it is possible to find sensors and/or actuators that have become faulty. As faults can result in abnormal operation/failure, effectively dealing with faulty situations for technical systems is an important specification to assess the global

\footnotetext{
*Corresponding author
}

performance of those systems. The objective of FTC is to maintain satisfactory performance for the controlled system even in the presence of faults.

In general, FTC is divided into passive (PFTC) and active (AFTC) (Blanke et al., 2006). The former deals with faults by using controller robustness while the latter handles faults after obtaining fault information by fault diagnosis techniques. PFTC is relatively easy to implement but has a restrictive fault-tolerant ability. Moreover, the larger the number of faults, the worse the control performance. Comparatively, AFTC 
is more flexible because it contains a fault diagnosis module to obtain real-time fault information. With the obtained fault information, AFTC can deal with faults more effectively. The fault diagnosis procedure embedded in an FTC scheme generally includes three steps: fault detection, fault isolation and fault estimation. Although AFTC is the topic of the proposed FTC scheme, this paper focuses more on the FD and FI tasks and assumes that actuator-fault magnitudes are known in advance. However, for FTC based on fault estimation and accommodation, the readers are referred to the works by Jiang and Chowdhury (2005), Jiang et al. (2006) or Xu et al. (2012). Additionally, due to a relatively low complexity and the ability of dealing with system constraints, MPC is chosen as the control strategy for the proposed scheme (Boskovic and Mehra, 2002; Maciejowski, 1999; Yang and Maciejowski, 2015). As an optimization-based method, robust MPC itself has a degree of the PFTC ability with respect to additive uncertainties (Borrelli et al., 2013).

In the work of Ocampo-Martinez et al. (2010), an actuator FTC scheme using feedback-gain control and invariant sets was proposed, where a bank of controllers were designed to handle faults in different actuators and the FDI task was implemented by using invariant set-based passive FDI methods. However, this scheme does not consider constraints on system variables and needs to wait until the residual has entered into its invariant set to isolate faults. In the work of Sun et al. (2008), a fault-tolerant model predictive control (FTMPC) scheme using the Kalman filter was proposed, which focused on the implementation of an FTMPC scheme without addressing in detail the features such as feasibility. Yetendje et al. (2011) presented an actuator FTMPC scheme with invariant set-based FDI, which had relatively low complexity because of the use of invariant sets for FDI. However, due to passive implementation of FDI, the set separation-based FDI condition is more conservative, which implies the loss of the potential FDI and FTC performance to some extent. The same authors extend the previous approach to the sensor case using a multisensor scheme (Yetendje et al., 2012). Raimondo et al. (2013) proposed an FTMPC scheme using set-membership FDI. Their work used an approach that combined passive FD and active FI, but employed a different implementing method. The active FI method proposed by Raimondo et al. (2013) can reduce the FI conservatism, at the cost of high computational complexity due to the requirement of computing fault-separating inputs on-line. Moreover, the scheme proposed by Raimondo et al. (2013) does not provide guaranteed FI conditions to check in advance whether or not the faults considered are isolable.

Since faults in actuators and sensors generally have different features, the current paper focuses on actuator faults by exploiting the potential of the proposed scheme. In particular, the objective of this paper is to propose a new scheme of actuator FTMPC not only to obtain less conservative FI and FI guarantees, but also to implement FTC with relatively low complexity. The proposed FTC scheme can also obtain a balance between the FI complexity and conservatism. In the scheme, FD is passively implemented with invariant sets while FI is actively carried out by using MPC and tubes that can isolate faults during the transition induced by faults.

The principle of active FI consists in adjusting the input-constraint set of the MPC controller to an off-line designed FI-oriented input set that can guarantee FI. In real time, whenever a fault is detected, the designed input set is used as the temporary input-constraint set of the MPC controller to implement FI during the transition. Moreover, since this input set is constructed off-line, guaranteed FI conditions can be verified off-line by using invariant sets and established on-line by the MPC controller for on-line FI guarantees. The proposed FTC scheme is shown in Fig. 1, where NS stands for nominal system and the subscript $k$ is only used to show that the discrete-time system is considered in this paper.

The advantages of the proposed scheme are twofold. First, a new actuator FTC scheme integrating MPC with set-based FDI to retain the advantages is proposed. Second, a new active FI strategy built upon tube-based MPC to obtain FI guarantees as well as a balance of FI conservatism and complexity is implemented. The work presented in this paper is inspired by our preliminary results (Xu et al., 2014).

The remainder of this paper is divided into five sections. Section 2, introduces the proposed FTC scheme. Section 3 presents the FDI strategy based on invariant sets and tubes. Section 4 introduces the FTC approach. In Section 5, a case study is used to show the effectiveness of the proposed scheme. Finally, Section 6 gives some conclusions on the approach.

Note that, in this paper, the inequalities are understood element-wise, $O, I$ and $\operatorname{diag}(\cdot)$ denote the zero, the identity and the diagonal matrices with suitable dimensions, respectively, $|\cdot|$ represents the element-wise absolute value, $\mathbb{B}^{r}$ is a box composed of $r$ unitary intervals, and $\oplus$ and $\ominus$ denote the Minkowski sum and Pontryagin difference, respectively.

\section{System description}

2.1. Plant model. It is assumed that the monitored system is described by a linear discrete time-invariant model including actuator faults, disturbances and noises:

$$
\begin{aligned}
x_{k+1} & =A x_{k}+B F u_{k}+\omega_{k}, \\
y_{k} & =C x_{k}+\eta_{k},
\end{aligned}
$$




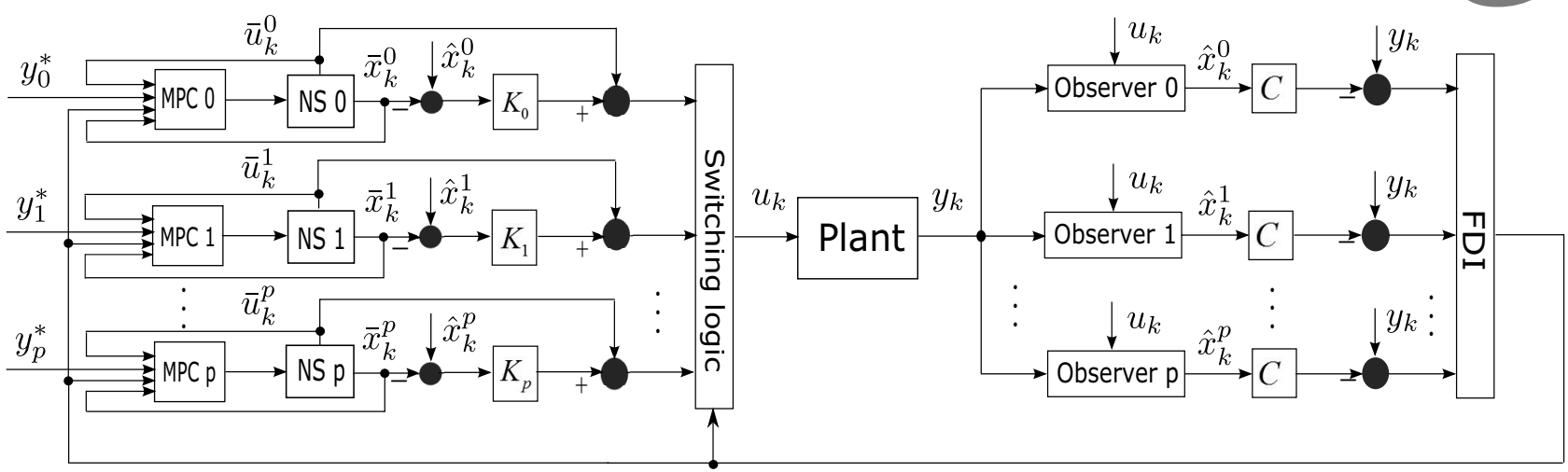

Fig. 1. Actuator FTMPC scheme.

where $A \in \mathbb{R}^{n \times n}, B \in \mathbb{R}^{n \times p}$ and $C \in \mathbb{R}^{q \times n}$ are constant parametric matrices, $x_{k} \in \mathbb{R}^{n}, u_{k} \in \mathbb{R}^{p}$ and $y_{k} \in \mathbb{R}^{q}$ are the state, input and output vectors at time instant $k$, respectively, $\omega_{k}$ and $\eta_{k}$ are respectively unknown process disturbances and measurement noise vectors, and $F$ is used to model actuator modes (healthy and faulty) which are important/critical for system performance/safety.

In (1), matrix $F$ incorporates the actuator modes considered. During real-time operation, a mode switching implies a change in the value of $F$ (i.e., fault occurrence or system recovery to the healthy situation). Moreover, $F$ is assumed to be constant for each mode but time-variant during the entire dynamical behavior where mode switchings are included.

Assumption 1. (Disturbances and noises) $\omega_{k}$ and $\eta_{k}$ are unknown but bounded by sets

$$
\begin{gathered}
W=\left\{\omega \in \mathbb{R}^{n}:\left|\omega-\omega^{c}\right| \leq \bar{\omega}\right\}, \\
V=\left\{\eta \in \mathbb{R}^{q}:\left|\eta-\eta^{c}\right| \leq \bar{\eta}\right\},
\end{gathered}
$$

respectively, where $\omega^{c}, \eta^{c}, \bar{\omega}$ and $\bar{\eta}$ are assumed to be known and constant vectors.

Assumption 2. (Faults considered) Single, abrupt and multiplicative faults are considered and the faults are assumed to be persistent, namely, the duration of the faults is longer than the detection and isolation time needed.

Under Assumption 2, it can be observed that $F$ can take $p+1$ different values, i.e., $F=F_{i}(i \in \mathbb{I}=$ $\{0,1,2, \ldots, p\}) . F_{0}$ is the identity matrix denoting the healthy actuator mode while $F_{i}(i \neq 0)$ modeling the $i$-th actuator-fault mode is denoted as

$$
F_{i}=\operatorname{diag}(1 \ldots 1 \stackrel{\substack{\downarrow \\ f}}{ } 1 \ldots 1)
$$

where $f_{i}$ is a scalar inside the interval $[0,1)$, which models the actuator-fault magnitude of the $i$-th actuator.
Notice that it is possible to extend the proposed FTMPC scheme to deal with sensor faults, multiple faults and additive faults. For example, if there are more elements, rather than a single element different from " 1 " in the fault-modeling matrix $F$, the proposed method can be used to handle multiple faults (see Reppa et al., 2015).

Assumption 3. (Stabilizability and detectability) The pairs $\left(A, B F_{i}\right)$ for all $i \in \mathbb{I}$ and $(A, C)$ are stabilizable and detectable, respectively.

In this scheme, the input and state constraints are taken into account, which are denoted as

$$
\begin{aligned}
& X=\left\{x \in \mathbb{R}^{n}:\left|x-x^{c}\right| \leq \bar{x}\right\}, \\
& U=\left\{u \in \mathbb{R}^{p}:\left|u-u^{c}\right| \leq \bar{u}\right\},
\end{aligned}
$$

respectively, where the vectors $x^{c}, u^{c}, \bar{x}$ and $\bar{u}$ are known and constant. $W, V, X$ and $U$ defined in (2) and (4) can be rewritten into zonotopes. Thus, in this paper, all set manipulations are implemented by zonotopes. The notion of zonotopes is given in Definition A1 in Appendix.

2.2. Output setpoints. It is mentioned that $p+1$ actuator modes are considered. Thus, the proposed FTMPC scheme should have $p+1$ different nominal models, each corresponding to one mode. For the $i$-th mode, the corresponding nominal model is given as

$$
\begin{aligned}
\bar{x}_{k+1}^{i} & =A \bar{x}_{k}^{i}+B F_{i} \bar{u}_{k}^{i}+\omega^{c}, \\
\bar{y}_{k}^{i} & =C \bar{x}_{k}^{i}+\eta^{c},
\end{aligned}
$$

where $\bar{u}_{k}, \bar{x}_{k}$ and $\bar{y}_{k}$ denote the nominal input, and state and output vectors, respectively. For simplicity, it is assumed that $\omega^{c}$ and $\eta^{c}$, representing the centers of the sets in $(2 a)$ and $(2 b)$, are zero vectors.

The control objective under the $i$-th mode is to regulate the output vector around a given setpoint $y_{i}^{*}$, i.e., in the absence of uncertainties,

$$
\lim _{k \rightarrow \infty}\left(y_{k}-y_{i}^{*}\right) \rightarrow 0 .
$$


In this paper, the model in (5) does not consider $\omega^{c}$ and $\eta^{c}$ for simplicity of the exposition. By using (5), a state-input setpoint pair $\left(x_{i}^{*}, u_{i}^{*}\right)$ corresponding to $y_{i}^{*}$ in the $i$-th mode can be computed by

$$
\left[\begin{array}{cc}
A-I & B F_{i} \\
C & O
\end{array}\right]\left[\begin{array}{l}
x_{i}^{*} \\
u_{i}^{*}
\end{array}\right]=\left[\begin{array}{c}
O \\
y_{i}^{*}
\end{array}\right]
$$

However, with no loss of generality, if $\omega^{c}$ and $\eta^{c}$ are non-zero, $\omega^{c}$ and $\eta^{c}$ can be added into (5a) and (5b), respectively.

Assumption 4. (State-input setpoint pair) For the $i$-th mode, (7) is solvable for all $i \in \mathbb{I}$.

Under Assumption 4 a state-input setpoint pair $\left(x_{i}^{*}, u_{i}^{*}\right)$ corresponding to $y_{i}^{*}$ can be obtained by solving (7) or degrading the expected performance (i.e., changing the output setpoint) in order to guarantee that (7) is solvable. For a given mode, (7) may have multiple solutions (i.e., the state-input setpoint pair may not be unique) or no solution. Thus, the designer should determine a satisfactory state-input setpoint pair according to particular requirements. Additionally, although the given output can be time-varying (i.e., the tracking problem), only the regulation problem is considered in this paper.

2.3. Observers and controllers. The tube-based MPC technique used in this scheme taps into the results of Mayne et al. (2006). As in their work, for each MPC controller, a state observer is designed. Thus, a bank of observers should be designed to match all modes, each observer matching one mode 1

Correspondingly, the observer matching the $j$-th $(j \in$ I) mode is designed as

$$
\begin{aligned}
\hat{x}_{k+1}^{j} & =\left(A-L_{j} C\right) \hat{x}_{k}^{j}+B F_{j} u_{k}+L_{j} y_{k}, \\
\hat{y}_{k}^{j} & =C \hat{x}_{k}^{j},
\end{aligned}
$$

where $\hat{x}_{k}^{j}$ and $\hat{y}_{k}^{j}$ are the estimated states and outputs, respectively, and $L_{j}$ is the $j$-th observer gain matrix that is selected to stabilize the observer dynamics (8), which is always possible under Assumption 3

In order to control the system under different actuator modes, a bank of tube-based output feedback MPC controllers are used, each corresponding to one mode. The nominal system corresponding to the $i$-th mode is obtained by neglecting $\omega_{k}$ and $\eta_{k}$ in (5).

\footnotetext{
${ }^{1}$ This is similar to the idea used in the multiple model adaptive estimation (MMAE) approach, where a bank of parallel Kalman filters, each with a different model, are employed. However, in the MMAE method (Hanlon and Maybeck, 2000), the hypothesis testing approach is used to determine which is the model with the highest likelihood to explain the input/output data.
}

According to Mayne et al. (2006), the control law of the $i$-th tube-based MPC controller is

$$
u_{k}=\bar{u}_{k}^{i}+K_{i}\left(\hat{x}_{k}^{i}-\bar{x}_{k}^{i}\right),
$$

where $K_{i}$ is the corresponding feedback-gain matrix.

\section{Fault detection and isolation}

3.1. System analysis. In the $i$-th mode, $F$ takes the value $F_{i}$ and the $i$-th tube-based MPC controller, the $i$-th state-input setpoint pair and the $i$-th observer are used in the closed-loop system. Moreover, the state estimation error of the $j$-th observer is defined as

$$
\tilde{x}_{k}^{i, j, i}=x_{k}-\hat{x}_{k}^{j} .
$$

Regarding the superscript notation $\tilde{x}_{k}^{i, j, i}$, the first index means the $i$-th system mode, the second denotes the $j$-th observer, and the third denotes that the $i$-th controller is currently selected for closed-loop operation. Thus, $\tilde{x}_{k}^{i, j, i}$ denotes the state estimation error of the $j$-th observer when the current closed-loop system is in the $i$-th mode and with the $i$-th MPC controller.

If $j \neq i$ in (10), the dynamics of $\tilde{x}_{k}^{i, j, i}$ can be derived by using (1), (8) and (9) as

$$
\begin{aligned}
\tilde{x}_{k+1}^{i, j, i}= & \left(A-L_{j} C\right) \tilde{x}_{k}^{i, j, i}+B\left(F_{i}-F_{j}\right) \bar{u}_{k}^{i}+\omega_{k} \\
& -L_{j} \eta_{k}+B\left(F_{i}-F_{j}\right) K_{i}\left(\hat{x}_{k}^{i}-\bar{x}_{k}^{i}\right),
\end{aligned}
$$

and the corresponding output-estimation error of the $j$-th observer can also be derived as

$$
\tilde{y}_{k}^{i, j, i}=y_{k}-\hat{y}_{k}^{j}=C \tilde{x}_{k}^{i, j, i}+\eta_{k} .
$$

Moreover, in the $i$-th mode, the term $\hat{x}_{k}^{i}-\bar{x}_{k}^{i}$ appearing in both (9) and (11) is denoted as

$$
e_{k}^{i, i, i}=\hat{x}_{k}^{i}-\bar{x}_{k}^{i} .
$$

Its dynamics can be derived by using (5) and (8) as

$$
e_{k+1}^{i, i, i}=\left(A+B F_{i} K_{i}\right) e_{k}^{i, i, i}+L_{i} C \tilde{x}_{k}^{i, i, i}+L_{i} \eta_{k},
$$

where $\tilde{x}_{k}^{i, i, i}$ corresponds to the case $j=i$ in 10 and its dynamics can be obtained from (11), i.e.,

$$
\tilde{x}_{k+1}^{i, i, i}=\left(A-L_{i} C\right) \tilde{x}_{k}^{i, i, i}+\left[\begin{array}{ll}
I & -L_{i}
\end{array}\right]\left[\begin{array}{c}
\omega_{k} \\
\eta_{k}
\end{array}\right] .
$$

Since $\omega_{k} \in W$ and $\eta_{k} \in V$, a robust positively invariant (RPI) set of $\tilde{x}_{k}^{i, i, i}$, denoted by $\tilde{X}^{i, i, i}$, can be constructed. In this paper, the notion of RPI sets and the method to construct those are based on the results of Kofman et al. (2007), Kolmanovsky and Gilbert (1998), and Olaru et al. (2010), which are given in Appendix.

As long as $\tilde{x}_{k^{*}}^{i, i, i} \in \tilde{X}^{i, i, i}$ holds, $\tilde{x}_{k}^{i, i, i} \in \tilde{X}^{i, i, i}$ always holds for all $k>k^{*}$. In the same way, considering $\tilde{x}_{k}^{i, i, i} \in$ 
$\tilde{X}^{i, i, i}$ and $\eta_{k} \in V$, an RPI set of $e_{k}^{i, i, i}$, denoted by $E^{i, i, i}$, can be constructed by using (14). For the $i$-th mode, if a fault is detected, an input set $\bar{U}_{f}^{i}$ is defined for FI analysis as

$\bar{U}_{f}^{i}=\left\{\bar{u}^{i} \in \mathbb{R}^{p}:\left|\bar{u}^{i}-\bar{u}_{f}^{i, c}\right| \leq \bar{u}_{f}^{i}, \bar{u}_{f}^{i, c} \in \mathbb{R}^{p}, \bar{u}_{f}^{i} \in \mathbb{R}^{p}\right\}$

which should be employed whenever a fault is detected we suppose that

$$
\bar{u}_{k}^{i} \in \bar{U}_{f}^{i},
$$

where $\bar{u}_{f}^{i, c}$ and $\bar{u}_{f}^{i}$ are constant and known vectors.

Remark 1. (Input-constraint set for FI) In this paper, an active FI strategy is proposed. The rationale behind the FI strategy is that, after FD, the input-constraint set of the $i$-th optimization problem corresponding to the $i$-th MPC controller is adjusted (to $\bar{U}_{f}^{i}$ ) to restrict inputs to values that can excite the plant to enable FI. Besides, $\bar{U}_{f}^{i}$ will only be used for FI when the system is in the $i$-th mode after FD. Moreover, $\bar{U}_{f}^{i}$ is different from the input-constraint set $U$. Likewise, in the proposed FI strategy, after a fault is isolated and simultaneously the system is reconfigured, $\bar{U}_{f}^{i}$ will not be used any longer. At this stage, $\bar{U}_{f}^{i}$ is introduced in order to help the readers understand the following proposed FI method. However, the detailed introduction of $\bar{U}_{f}^{i}$ will be given in Section 4

Similarly, for $e_{k}^{i, i, i} \in E^{i, i, i}$ and $\bar{u}_{k}^{i} \in \bar{U}_{f}^{i}$ in (11), an RPI set of $\tilde{x}_{k}^{i, j, i}$, denoted by $\tilde{X}^{i, j, i}$, can be determined. Furthermore, the set of the corresponding output-estimation error is

$$
\tilde{Y}^{i, j, i}=C \tilde{X}^{i, j, i} \oplus V .
$$

For the particular case when $j=i$, the output-estimation-error set $\tilde{Y}^{i, i, i}$ corresponding to $\tilde{X}^{i, i, i}$ can also be determined and used for FDI. Generally, the RPI sets $\tilde{X}^{i, i, i}, E^{i, i, i}$ and $\tilde{X}^{i, j, i}$ should be as small as possible, being ideally tight approximations of the minimal RPI set.

3.2. Fault detection. The FD approach used in this paper is a passive one based on invariant sets, which can simplify the FD task into only testing whether or not the residual is inside its corresponding invariant set. The advantage of the FD method used consists in its low computational complexity.

Considering (11) and (14), since $\omega_{k} \in W$ and $\eta_{k} \in$ $V$, if $\bar{u}_{k}^{i} \in \bar{U}_{f}^{i}$ always holds, it can be observed that, as long as $e_{k}^{i, i, i} \in E^{i, i, i}$ holds, $\tilde{x}_{k}^{i, j, i} \in \tilde{X}^{i, j, i}(j \neq i)$ can always hold. However, as for (15), it can be seen that $\tilde{X}^{i, i, i}$ is independent of the effect of $e_{k}^{i, i, i}$ and $\bar{u}_{k}^{i}$ while $E^{i, i, i}$ is dependent of $\tilde{X}^{i, i, i}$. Thus, theoretically, the most convenient way to detect faults is to test the inclusion $\tilde{x}_{k}^{i, i, i} \in \tilde{X}^{i, i, i}$ 2 But, practically, since $\tilde{x}_{k}^{i, i, i}$ is not obtainable, instead, only the signal $\tilde{y}_{k}^{i, i, i}$ can be used. Thus, the first criterion for FD is to test whether or not

$$
\tilde{y}_{k}^{i, i, i} \in \tilde{Y}^{i, i, i}
$$

is violated in real time. If a violation of (17) is detected, this means that a fault has occurred. Otherwise, it is considered that the system still operates in the $i$-th mode.

Although (17) can be used for FD, if only 17) is applied, the fault sensitivity of the proposed FTC scheme will not be fully exploited. The reason is due to the fact that, even though (17) holds, it cannot be guaranteed that the inclusion $\tilde{x}_{k}^{i, i, i} \in \tilde{X}^{i, i, i}$ holds, too, which means that the detection of $\tilde{y}_{k}^{i, i, i} \in \tilde{Y}^{i, i, i}$ has a different fault sensitivity from that of $\tilde{x}_{k}^{i, i, i} \in \tilde{X}^{i, i, i}$. In this case, it is necessary to consider the second FD criterion to describe this situation, i.e., to test whether or not

$$
e_{k}^{i, i, i} \in E^{i, i, i}
$$

is violated in real time. If (18) is violated, this also implies that a fault has occurred. Note that, as mentioned before, the FD criterion (18) can indirectly describe the inclusions corresponding to the other observers, i.e.,

$$
\tilde{y}_{k}^{i, j, i} \in \tilde{Y}^{i, j, i}, \quad j \neq i .
$$

Thus, the FD strategy of this proposed FTC scheme is to use both (17) and (18). As long as either of them is violated, this implies that the system has become faulty.

Remark 2. (Fault detection) Simultaneous use of the criteria (17) and (18) means that the system information captured by all the observers has been used for FD. Additionally, for the proposed FD strategy, even though some faults occur, it is possible that the FD criteria (17) and (18) are not violated. This means that these faults cannot be detected and will not actively be tolerated under the framework of this proposed active FTC scheme. Instead, they can only be tolerated to some extent by the PFTC ability of the proposed scheme.

\subsection{Fault isolation.}

3.3.1. Behavior after faults. In the scheme, the FI task is started up after a fault is detected by the proposed FD strategy. With no loss of generality, it is assumed that the $l$-th $(l \neq i)$ fault occurs, i.e., after that, the system

\footnotetext{
${ }^{2}$ Under the framework of the proposed FTC scheme, mode switching has several different cases including the situations from the healthy mode to a faulty mode, from a faulty mode to another faulty mode, and from a faulty mode to the healthy mode. However, with no loss of generality, the terms and/or concepts fault, fault occurrence, fault detection and fault isolation are used in this paper to generally mean mode, mode switching, mode-switching detection and mode-switching isolation, respectively.
} 
mode changes from $i$ to $l$. Although the mode changes from $i$ to $l$, before the fault is isolated and the system is reconfigured, the closed-loop system structure will not change yet, which implies that the closed-loop system is still composed of the same controller and observer during the FI phase.

According to (1), (5), (8) and (9), when the $l$-th fault occurs, the state estimation error of the $j$-th observer changes from $\tilde{x}_{k}^{i, j, i}$ to $\tilde{x}_{k}^{l, j, i}$ with the dynamics

$$
\begin{aligned}
\tilde{x}_{k+1}^{l, j, i}= & \left(A-L_{j} C\right) \tilde{x}_{k}^{l, j, i}+B\left(F_{l}-F_{j}\right) \bar{u}_{k}^{i}+\omega_{k} \\
& -L_{j} \eta_{k}+B\left(F_{l}-F_{j}\right) K_{i} e_{k}^{l, i, i}
\end{aligned}
$$

and $e_{k}^{i, i, i}$ in (14) changes to $e_{k}^{l, i, i}$ with the dynamics

$$
e_{k+1}^{l, i, i}=\left(A+B F_{i} K_{i}\right) e_{k}^{l, i, i}+L_{i} C \tilde{x}_{k}^{l, i, i}+L_{i} \eta_{k} .
$$

In order to collect all the available system-operating information for fault diagnosis after the $l$-th fault from the $i$-th mode, a vector is defined as

$$
\xi_{k}^{i \rightarrow l}=\left[\begin{array}{llllll}
\tilde{x}_{k}^{l, 0, i} & \cdots & \tilde{x}_{k}^{l, i, i} & \cdots & \tilde{x}_{k}^{l, p, i} & e_{k}^{l, i, i}
\end{array}\right]^{T} .
$$

According to 20) and (21), the dynamics of $\xi_{k}^{i \rightarrow l}$ can be obtained as

$$
\xi_{k+1}^{i \rightarrow l}=A_{i \rightarrow l} \xi_{k}^{i \rightarrow l}+B_{i \rightarrow l} \bar{u}_{k}^{i}+E_{i \rightarrow l}^{\omega} \omega_{k}+E_{i \rightarrow l}^{\eta} \eta_{k},
$$

where

$$
\begin{aligned}
A_{i \rightarrow l}= & {\left[\begin{array}{cccc}
A-L_{0} C & O & \cdots & O \\
\vdots & \vdots & \cdots & \vdots \\
O & A-L_{i} C & \cdots & O \\
\vdots & \vdots & \cdots & \vdots \\
O & O & \cdots & A-L_{p} C \\
O & L_{i} C & \cdots & O \\
B\left(F_{l}-F_{0}\right) K_{i} \\
\vdots \\
B\left(F_{l}-F_{i}\right) K_{i} \\
\vdots \\
B\left(F_{l}-F_{p}\right) K_{i} \\
A+B F_{i} K_{i}
\end{array}\right] }
\end{aligned}
$$

$$
\begin{aligned}
& B_{i \rightarrow l}= {\left[\begin{array}{c}
B\left(F_{l}-F_{0}\right) \\
\vdots \\
B\left(F_{l}-F_{i}\right) \\
\vdots \\
B\left(F_{l}-F_{p}\right) \\
O
\end{array}\right], } \\
& E_{i \rightarrow l}^{\omega}=\left[\begin{array}{c}
I \\
\vdots \\
I \\
\vdots \\
I \\
O
\end{array}\right], \quad E_{i \rightarrow l}^{\eta}=\left[\begin{array}{c}
-L_{0} \\
\vdots \\
-L_{i} \\
\vdots \\
-L_{p} \\
L_{i}
\end{array}\right] .
\end{aligned}
$$

Remark 3. (Stability) Under Assumption 3 , the observer and feedback gains $L_{0}, L_{1}, \ldots, L_{p}$ and $F_{0}, F_{1}, \ldots, F_{p}$ can be designed to make $A_{i \rightarrow l}$ a Schur matrix for all $i, l \in \mathbb{I}$. With (22), the closed-loop system can be stable by designing the observer and feedback gains and the parameters of open-loop optimization problem of the tube-based MPC controller (see Mayne et al., 2006).

Furthermore, as for $\bar{u}_{k}^{i} \in \bar{U}_{f}^{i}, \omega_{k} \in W$ and $\eta_{k} \in V$, an RPI set of $\xi_{k}^{i \rightarrow l}$ can be constructed, which is denoted by $\Xi^{i \rightarrow l}$. By projecting $\Xi^{i \rightarrow l}$ towards the component space, an RPI set of each component of $\xi_{k}^{i \rightarrow l}$ can be obtained. For example, an RPI set (denoted by $\tilde{X}^{l, j, i}$ ) of $\tilde{x}_{k}^{l, j, i}$ can be obtained by projecting $\Xi^{i \rightarrow l}$ to the space of $\tilde{x}_{k}^{l, j, i}$. Similarly, an RPI set (denoted by $E^{l, i, i}$ ) of $e_{k}^{l, i, i}$ can be constructed. This implies that, after the $l$-th fault, $\tilde{x}_{k}^{l, j, i}$ and $e_{k}^{l, i, i}$ will converge into $\tilde{X}^{l, j, i}$ and $E^{l, i, i}$, respectively. Moreover, with (2b), the set of the corresponding output-estimation error can be obtained as

$$
\tilde{Y}^{l, j, i}=C \tilde{X}^{l, j, i} \oplus V .
$$

When the system mode switches from $i$ to $l$, all sets of output-estimation errors can be constructed, which are listed in Table 1 Note that, in Table 1 each row excluding the $i$-th one corresponds to one candidate mode after the mode switching from the $i$-th one.

3.3.2. Residual tubes. Generally, the residual is defined as a signal sensitive to faults and with a manageable magnitude. In this FTC scheme, the output-estimation errors are defined as residual signals. The dynamics of $\tilde{x}^{l, l, i}$ extracted from (22) are used for FI implementation, which has the form

$$
\tilde{x}_{k+1}^{l, l, i}=\left(A-L_{l} C\right) \tilde{x}_{k}^{l, l, i}+\omega_{k}-L_{l} \eta_{k},
$$

while $\tilde{x}^{l, j, i}(j \neq l)$ will not be used for direct FI implementation but for the establishment of guaranteed FI conditions. By using $W$ and $V$ to replace $\omega_{k}$ and 
Table 1. Sets of output-estimation errors.

\begin{tabular}{|c|c|c|c|c|c|}
\hline & Observer 0 & $\cdots$ & Observer $i$ & $\cdots$ & Observer $p$ \\
\hline \hline Mode 0 & $\tilde{Y}^{0,0, i}$ & $\cdots$ & $\tilde{Y}^{0, i, i}$ & $\cdots$ & $\tilde{Y}^{0, p, i}$ \\
$\vdots$ & $\vdots$ & $\cdots$ & $\vdots$ & $\cdots$ & $\vdots$ \\
Mode $i$ & $\tilde{Y}^{i, 0, i}$ & $\cdots$ & $\tilde{Y}^{i, i, i}$ & $\cdots$ & $\tilde{Y}^{i, p, i}$ \\
$\vdots$ & $\vdots$ & $\cdots$ & $\vdots$ & $\cdots$ & $\vdots$ \\
Mode $p$ & $\tilde{Y}^{p, 0, i}$ & $\cdots$ & $\tilde{Y}^{p, i, i}$ & $\cdots$ & $\tilde{Y}^{p, p, i}$ \\
\hline
\end{tabular}

$\eta_{k}$, the set-based description of $\tilde{x}_{k}^{l, l, i}$ and $\tilde{y}_{k}^{l, l, i}$ can be obtained as

$$
\begin{aligned}
\tilde{X}_{k+1}^{l, l, i} & =\left(A-L_{l} C\right) \tilde{X}_{k}^{l, l, i} \oplus W \oplus\left(-L_{l} V\right), \\
\tilde{Y}_{k}^{l, l, i} & =C \tilde{X}_{k}^{l, l, i} \oplus V .
\end{aligned}
$$

Proposition 1. (Estimation-error tubes) Given that the $l$ th $(l \neq i)$ fault occurs when the system is in the $i$-th mode and the state estimation error $\tilde{x}_{k *}^{l, l, i}$ of the l-th observer is bounded by a set $\tilde{X}_{k *}^{l, l, i}$ at time instant $k *$, if $\tilde{X}_{k *}^{l, l, i}$ is used to initialize (25) to generate tubes, $\tilde{x}_{k}^{l, l, i} \in \tilde{X}_{k}^{l, l, i}$ and $\tilde{y}_{k}^{l, l, i} \in \tilde{Y}_{k}^{l, l, i}$ will hold for all $k \geq k *$.

Proof. Since 25a considers the worst case of the uncertain factors $\omega_{k}$ and $\eta_{k}$ in (24), if $\tilde{x}_{k *}^{l, l, i} \in \tilde{X}_{k *}^{l, l, i}$ holds at time instant $k *$, this implies that $\tilde{x}_{k}^{l, l, i} \in \tilde{X}_{k}^{l, l, i}$ and $\tilde{y}_{k}^{l, l, i} \in \tilde{Y}_{k}^{l, l, i}$ will always hold for all $k \geq k *$.

It is assumed that the $l$-th fault is detected at time instant $k_{d}$ when the system is in the $i$-th mode. If an initial set is used to initialize 25a at time instant $k_{d}$, the tubes corresponding to the state and output estimation errors generated by (25) can be denoted as

$$
\begin{aligned}
\tilde{\mathbb{T}}_{k_{d}}^{x, l, l, i} & =\left\{\tilde{X}_{k_{d}}^{l, l, i}, \tilde{X}_{k_{d}+1}^{l, l, i}, \tilde{X}_{k_{d}+2}^{l, l, i}, \ldots\right\}, \\
\tilde{\mathbb{T}}_{k_{d}}^{y, l, l, i} & =\left\{\tilde{Y}_{k_{d}}^{l, l, i}, \tilde{Y}_{k_{d}+1}^{l, l, i}, \tilde{Y}_{k_{d}+2}^{l, l, i}, \ldots\right\} .
\end{aligned}
$$

That initial set is used to initialize the dynamics (25) to generate tubes for FI and introduced here for the discussion of the FI method. A detailed construction method for set initialization will be presented in Section 3.3.4.

When the system is in the $i$-th mode, a violation of (17) or 18) implies that a mode changing from $i$ to another unknown mode has occurred (this unknown mode is denoted as $f \in \mathbb{I} \backslash\{i\}$ ), i.e., there are $p$ mode candidates except for the $i$-th one. Thus, for FI, all the $p$ output-estimation error tubes $\tilde{\mathbb{T}}_{k_{d}}^{y, l, l, i}(l \in \mathbb{I} \backslash\{i\})$ have to be obtained. At time instant $k_{d}$, the proposed FI algorithm generates $p$ output-estimation-error tubes $\tilde{\mathbb{T}}_{k_{d}}^{y, l, l, i}(l \in \mathbb{I} \backslash$ $\{i\}$ ), each corresponding to a candidate mode. Moreover, for the $p$ corresponding observers, as long as

$$
\tilde{x}_{k_{d}}^{f, l, i} \subseteq \tilde{X}_{k_{d}}^{l, l, i}, \quad f, l \in \mathbb{I} \backslash\{i\}
$$

are guaranteed at the FD time such that

$$
\tilde{y}_{k_{d}}^{f, l, i} \subseteq \tilde{Y}_{k_{d}}^{l, l, i} .
$$

Therefore, this implies that, among the $p$ generated output-estimation-error tubes after FD, there exists at least one tube (here it is assumed that this tube corresponds to the $m$-th actuator mode) that can always satisfy

$$
\tilde{y}_{k}^{f, m, i} \subseteq \tilde{Y}_{k}^{m, m, i}, \quad k \geq k_{d}, \quad m \in \mathbb{I} \backslash\{i\} .
$$

If the fault is indexed by $l$ (i.e., $f=l$ ) and (27) is satisfied for all $k \geq k_{d}, \tilde{\mathbb{T}}_{k_{d}}^{y, l, l, i}$ can always satisfy $\tilde{y}_{k}^{f, l, i} \subseteq$ $\tilde{Y}_{k}^{l, l, i}$. This implies that the fault will be indicated by one of the $p$ tubes that can always satisfy 29.

3.3.3. Fault isolation approach. In order to isolate a fault, it has to guarantee that one and only one tube can always satisfy its corresponding inclusion (29) after FD and then the fault can be indicated by the index of this tube. Based on this idea, guaranteed FI conditions are established in Proposition 2

Proposition 2. (Guaranteed FI conditions) When the system is in the $i$-th mode, for any observer out of the $p+1$ observers (assume that it is indexed by $j$ ), if all the $p+1$ output-estimation-error sets corresponding to this observer (i.e., the $p+1$ sets in the $j$-th column of Table 1 can satisfy

$$
\tilde{Y}^{j, j, i} \cap \bigcup_{l=0}^{p} \tilde{Y}^{l, j, i}=\emptyset, \quad l \neq j, \quad i, j, l \in \mathbb{I},
$$

once a mode changing from the $i$-th mode to another considered mode is detected at time instant $k_{d}$, this mode can be isolated during the transition induced by the mode changing by searching the output-estimation-error tube that satisfies 29) for all $k \geq k_{d}$.

Proof. As concluded, $\tilde{\mathbb{T}}_{k_{d}}^{y, j, j, i}$ will converge to $\tilde{Y}^{j, j, i}$. If (30) holds, $\tilde{\mathbb{T}}_{k_{d}}^{y, j, j, i}$ is able to confine the output-estimation error $\tilde{y}_{k}^{l, j, i}$ only under the condition $l=j$. If $l \neq j$, at the first several steps, $\tilde{\mathbb{T}}_{k_{d}}^{y, j, j, i}$ is able to confine $\tilde{y}_{k}^{l, j, i}$ due to the 
initialization condition 27]. But, as $\tilde{\mathbb{T}}_{k_{d}}^{y, j, j, i}$ approaches $\tilde{Y}^{j, j, i}, \tilde{y}_{k}^{l, j, i}$ diverges from $\tilde{\mathbb{T}}_{k_{d}}^{y, j, j, i}$. This implies that, under the condition (30), by searching the tube that is always able to confine $\tilde{y}_{k}^{l, j, i}$ after FD, the fault can be isolated.

3.3.4. Construction of initial sets. As mentioned in (26), one of the key points of the proposed FI strategy consists in constructing the initial sets of state estimation errors, which satisfy (27) at time instant $k_{d}$ to initialize (25) in order to generate output-estimation-error tubes. For the $j$-th observer, according to $(12)$, it can obtain

$$
C \tilde{x}_{k_{d}}^{i, j, i} \in\left\{\tilde{y}_{k_{d}}^{i, j, i}\right\} \oplus(-V) .
$$

In (31), since $\tilde{y}_{k_{d}}^{i, j, i}$ can be obtained in real time, it is always possible to construct a zonotopic set containing $\tilde{x}_{k_{d}}^{i, j, i}$ at the FD time. In the work of Alamo et al. (2005), a method computing a zonotope containing the intersection of a strip and a zonotope is given in Property A4 Based on this method, a zonotope containing $\tilde{x}_{k_{d}}^{i, j, i}$ can be constructed by considering (31) composed of $q$ inequalities (i.e., strips). Besides, the method proposed by Le et al. (2013) can also be used to construct a zonotopic set containing $\tilde{x}_{k_{d}}^{i, j, i}$. This method can compute a zonotopic approximation of the intersection of a zonotope and a polytope. With this method, (31) is regarded as a whole that describes a polytope to construct an initial zonotope, which can be seen in Property A5

Remark 4. (Construction of initial sets) If $C$ is invertible, a set bounding $\tilde{x}_{k_{d}}^{i, j, i}$ can be directly obtained by (31) with the inverse of $C$. If $C$ is not invertible, an initial zonotope to bound $\tilde{x}_{k_{d}}^{i, j, i}$ can be obtained by the method in Propositions A4 or A5. In the second case, it may need to give a zonotopic starting set for the methods in Propositions A4 and A5 and this set can be designed according to the physical constraints of the system.

According to (31), it can be observed that, for the $j$-th observer, the expression of (31) is independent of system mode changing. This means that (31) can always be used to construct a set to bound the state estimation error of the $j$-th observer in any mode. Since $X, U, W$ and $V$ can be rewritten as zonotopes, from the computational point of view, all tubes are generated by using zonotopes.

\section{Fault-tolerant control}

4.1. Steady-state behaviors. In the proposed FTC scheme, system operation is divided into the transient-state and steady-state phases. The steady-state operation is observed when all relevant system signals corresponding to a system mode are inside their corresponding bounding sets. Comparatively, the transient-state operation describes the operating process between fault occurrence and the steady-state operation of the mode corresponding to this fault. In this paper, these two operations will be discussed, separately. This subsection focuses on system behavior during the steady-state operation.

At steady state of the $i$-th mode, the tube-based MPC technique proposed by Mayne et al. (2006) is adopted to implement FTC, and the control law of the $i$-th one is given in (9). For the tube-based MPC controller (9), the key part $\bar{u}_{k}^{i}$ is the open-loop optimization problem based on the $i$-th nominal system as in (5).

$X$ and $U$ are hard system constraints that imply indirect constraints on the nominal system-based open-loop optimization problem. In the $i$-th mode, the indirect input constraint is computed via (9), i.e.,

$$
u_{k}=\bar{u}_{k}^{i}+K_{i} e_{k}^{i, i, i}
$$

As in Section 3.1, at a steady state of the $i$-th mode,

$$
e_{k}^{i, i, i} \in E^{i, i, i}
$$

should hold. Thus, the input-constraint set of the open-loop optimization problem can be obtained as

$$
\bar{u}_{k}^{i} \in \bar{U}^{i}=U \ominus K_{i} E^{i, i, i} .
$$

Additionally, taking

$$
x_{k}=\bar{x}_{k}^{i}+e_{k}^{i, i, i}+\tilde{x}_{k}^{i, i, i}
$$

into account, the hard state-constraint set for steady-state functioning can be described as

$$
\bar{x}_{k}^{i} \in \bar{X}^{i}=X \ominus\left(E^{i, i, i} \oplus \tilde{X}^{i, i, i}\right) .
$$

Assumption 5. (Indirect constraint sets) In the $i$-th mode, $\bar{X}^{i}$ and $\bar{U}^{i}$ are non-empty for all $i \in \mathbb{I}$.

The non-emptiness of $\bar{X}^{i}$ and $\bar{U}^{i}$ is the precondition for using the tube-based MPC technique. Assumption 5 is a well-known and accepted condition in the field. Under Assumption 5, the open-loop optimization problem of the $i$-th tube-based MPC controller, based on the $i$-th nominal system (5), has the following form:

$$
\begin{gathered}
J_{k}=\min _{\overline{\mathbf{u}}^{i}} \sum_{j=0}^{N-1}\left\|\left(\bar{x}_{k+j \mid k}^{i}-x_{i}^{*}\right)\right\|_{Q_{i}}^{2}+\left\|\left(\bar{u}_{k+j \mid k}^{i}-u_{i}^{*}\right)\right\|_{R_{i}}^{2} \\
+\left\|\left(\bar{x}_{k+N \mid k}^{i}-x_{i}^{*}\right)\right\|_{P_{i}}^{2} \\
\text { subject to } \quad \bar{x}_{k+j \mid k}^{i} \in \bar{X}^{i}, \\
\bar{u}_{k+j \mid k}^{i} \in \bar{U}^{i}, \\
\bar{x}_{k+N \mid k}^{i} \in \bar{X}_{T}^{i}, \\
\bar{x}_{k \mid k}^{i}=\bar{x}_{k}^{i},
\end{gathered}
$$

where

$$
\overline{\mathbf{u}}^{\mathbf{i}}=\left[\bar{u}_{k \mid k}^{i}, \bar{u}_{k+1 \mid k}^{i}, \cdots, \bar{u}_{k+N-1 \mid k}^{i}\right]
$$


is the optimized control sequence over the horizon $N$, $Q_{i} R_{i}$ and $P_{i}$ are positive-definite matrices, and $\bar{X}_{T}^{i}$ is the corresponding terminal state constraint set. The purpose of adding the terminal constraint in 34 is for the feasibility and stability. In (34), $\bar{X}_{T}^{i}$ is defined as the maximal control invariant (MCI) set of the $i$-th nominal system corresponding to the nominal constraint sets $\bar{X}^{i}$ and $\bar{U}^{i}$ such that the $i$-th tube-based MPC controller is feasible (see Definition A6 in Appendix for the MCI sets). As mentioned in Remark 3, the tube-based MPC controller can be designed to make the closed-loop system stable; see the works of Borrelli et al. (2013) and Mayne et al. (2006) for details of tube-based MPC.

4.2. Transient-state behavior before FD. As mentioned before, after fault occurrence, the system quits from the steady-state operation and enters the transient-state operation. Different from the steady-state operation of the $i$-th mode, fault occurrence implies that the system mode changes from the $i$-th one to another one that will be denoted by an index $l(l \neq i)$.

In order to analyze the transient-state behavior induced by a fault, the transient-state operation is divided into three different phases. The first one starts from the occurrence till detection of the fault, the second starts from the detection to isolation of the fault and the third begins from system reconfiguration to the steady-state operation of the $l$-th mode. Taking into account that the second and third phases of the transition correspond to the FI task, this subsection only focuses on the first-phase transition while the other two transient-state phases will be discussed in the next subsection.

Remark 5. (After-fault behavior) When the system is in the $i$-th mode at the beginning, after the $l$-th fault, $\tilde{y}_{k}^{i, i, i}$ and $e_{k}^{i, i, i}$ will change into $\tilde{y}_{k}^{l, i, i}$ and $e_{k}^{l, i, i}$, respectively.

During the first phase of the transition, even though the $l$-th fault has occurred, the FD criteria (17) and (18) still hold, i.e.,

$$
\tilde{y}_{k}^{l, i, i} \in \tilde{Y}^{i, i, i}
$$

and

$$
e_{k}^{l, i, i} \in E^{i, i, i} .
$$

Although the FD criteria (17) and (18) still hold during the first phase of the transition, it cannot be guaranteed that

$$
\tilde{x}_{k}^{l, i, i} \in \tilde{X}^{i, i, i}
$$

can still hold, which can be observed from 20 and (21). This problem is inevitable. Because the satisfaction of (35) cannot be guaranteed, during this transient-state phase, the state constraint

$$
x_{k}=\bar{x}_{k}^{i}+e_{k}^{l, i, i}+\tilde{x}_{k}^{l, i, i} \in X
$$

may be violated. However, notice that, during the first phase of the transition, the input constraint

$$
u_{k}=\bar{u}_{k}^{i}+K_{i} e_{k}^{l, i, i} \in U
$$

always holds under the satisfaction of Assumption 5 and $e_{k}^{l, i, i} \in E^{i, i, i}$. As has been mentioned, since the problem indicated in (35) is inevitable, the satisfaction of the state constraint has to be assumed during this phase.

Assumption 6. (First-phase transition) During the first-phase transition, the inclusion $x_{k}=\bar{x}_{k}^{i}+e_{k}^{l, i, i}+$ $\tilde{x}_{k}^{l, i, i} \in X$ always holds.

Since the open-loop optimization problem in 34 is not affected by the real system, its feasibility can always be preserved during the first phase of the transition. Moreover, during this phase, the closed-loop system is still composed of the same elements with the $i$-th faultfree mode. Although the $l$-th fault has occurred, the process assumes that the system still operates in the $i$-th mode as long as both state and input constraints are satisfied.

4.3. Transient-state behavior during FI. The active FI task corresponds to the second phase of the transition. During this phase, it is already known that a fault has occurred in the system. Thus, the most important objective is to isolate the fault. The basic FI principle here is to directly change the input-constraint set of the $i$-th open-loop optimization problem on the $i$-th nominal system to indirectly change the input set of the plant to force the satisfaction of the proposed FI conditions by means of the constraint-handling ability of the open-loop MPC optimization problem behind the MPC controller. In this way, the plant input vector can be confined into a predefined set $U_{f}^{i}$ to excite the system and to obtain more system-operating information for FI implementation. Note that $U_{f}^{i}$ for active FI has already been briefly introduced in Remark 1

As observed from (22) and (23), when the system mode changes from $i$ to $l$, the sets of the state and output estimation errors are determined by the sets of $\bar{u}_{k}^{i}$, $\omega_{k}$ and $\eta_{k}$, and the fault magnitudes if it is considered that the observer and feedback gains have already been designed. Without explicitly considering the observer and feedback gains, a function is used to describe the sets of the output-estimation errors to help the readers understand the proposed FI approach, i.e.,

$$
\tilde{Y}^{l, j, i}=f^{i \rightarrow l}\left(\bar{U}_{f}^{i}, W, V\right), \quad j \neq l,
$$

which implies that whether or not the guaranteed FI conditions in Proposition 2 hold depends on adjusting the set of the nominal inputs $\bar{u}_{k}^{i}$. Note that $\tilde{Y}^{l, l, i}$ is determined by $W$ and $V$ and is free from the effect of $\bar{U}_{f}^{i}$. 
Assumption 7. (Input-constraint set) In the $i$-th mode, for all $i \in \mathbb{I}$, there exists an input set $\bar{U}_{f}^{i}$ such that the FI conditions proposed in Proposition 2 are satisfied.

Thus, under Assumption 7, at the time instant when a switching from the mode $i$ to $l$ is detected, if $\bar{u}_{k}^{i}$ can always be confined inside the FI input set $\bar{U}_{f}^{i}$ by the open-loop optimisation problem of the $i$-th MPC controller, the FI conditions in Proposition 2 can be forced to hold on-line by $\bar{U}_{f}^{i}$, and then the FI approach proposed in Section 3.3 can be used to isolate the fault. Thus, when the system is in the $i$-th mode, the tube-based MPC controller has two objectives:

- Steady-state operation (including the first-phase transition): no fault is detected and the main task is to achieve system performance. Thus, in order to make full use of the potential performance of the system, the input-constraint set $\bar{U}^{i}$ is used for the $i$-th open-loop optimization problem.

- Transient-state operation (only the second phase): a fault is detected and the main task is to isolate and reconfigure the system to obtain satisfactory performance even in the presence of the fault. During this stage, the proposed FI approach actively adjusts the input-constraint set of the $i$-th open-loop optimization problem from $\bar{U}^{i}$ to $\bar{U}_{f}^{i}$ at the FD time $k_{d}$ to establish the FI conditions on-line, which is the proposed active FI strategy.

During the second phase of the transition (i.e., the FI process), in addition to guaranteeing the satisfaction of the FI conditions, the feasibility, stability and constraint satisfaction of the controller and system should also be considered. The optimization problem 34 is updated by directly using the nominal state from the nominal prediction model. The nominal states are generated by the nominal prediction model free from the effect of the real system. Thus, as long as the $i$-th open-loop optimization problem can be designed to be feasible, the feasibility feature of the optimization can be preserved during the whole FI process if the constraints $X$ and $U$ are not considered. Since the set of the nominal input vectors of the $i$-th nominal system is adjusted for FI, the feasibility of the $i$-th optimization problem should be preserved by using a new pair of constraint sets.

Thus, during the FI process, except that the input constraint of (34) is switched from $\bar{U}^{i}$ to $\bar{U}_{f}^{i}$ to establish the FI conditions on-line, the state and terminal constraints are accordingly switched from $\bar{X}^{i}$ to $\bar{X}_{f}^{i}$ and $\bar{X}_{T}^{i}$ to $\bar{X}_{f}^{i}$, respectively. The set $\bar{X}_{f}^{i}$ is the state-constraint set of (34) for the FI process and $\bar{X}_{f_{T}}^{i}$ is a control invariant (CI) set of the $i$-th nominal system corresponding to $\bar{u}_{k}^{i} \in \bar{U}_{f}^{i}$ and $\bar{x}_{k}^{i} \in \bar{X}_{f}^{i}$. The sets $\bar{U}_{f}^{i}$ and
$\bar{X}_{f}^{i}$ are a pair of designing parameters used to guarantee FI and constraint satisfaction in this FTC scheme.

Remark 6. (Transient constraint satisfaction) During the FI process, from mode $i$ to $l, \bar{U}_{f}^{i} \oplus K_{i} e_{k}^{l, i, i} \in U$ and $\bar{X}_{f}^{i} \oplus e_{k}^{l, i, i} \oplus \tilde{x}_{k}^{l, i, i} \in X$ should hold such that the hard input and state constraints are not violated, which is the precondition of the proposed FTC scheme and is used to ensure the availability of the tube-based MPC technique. The satisfaction of this condition can be affected by system dynamics, faults, $\bar{U}_{f}^{i}$ and $\bar{X}_{f}^{i}$. This means that a proper pair of $\bar{U}_{f}^{i}$, and $\bar{X}_{f}^{i}$ should be designed to guarantee the effectiveness of the proposed FI strategy.

Based on the explanation of Remark 6, in order to ensure the availability of the proposed FTC scheme, Assumption 8 is further made.

Assumption 8. (Transient constraint sets) There exists a pair of $\bar{U}_{f}^{i}$ and $\bar{X}_{f}^{i}$ such that the constraints $u_{k} \in U$ and $x_{k} \in X$ are not violated during the whole FI phase.

Notice that the selection of the pair $\left(u_{k} \in U\right.$ and $x_{k} \in X$ ) plays an important role in the proposed FTC scheme. Since the methodological procedure of selecting that pair is out of the scope of this paper, $\bar{U}_{f}^{i}$ and $\bar{X}_{f}^{i}$ have been selected by trial and error towards a suitable operation of the proposed approach. During the FI task, in addition to constraint satisfaction, the feasibility and stability of the $i$-th open-loop optimization problem with a new pair of constraint sets should be guaranteed as well. Based on the optimization (34), to guarantee its feasibility, the nominal states generated from the nominal system internal model inside its terminal state constraints should always be confined in the MCI set. Thus, at the FD time $k_{d}$, when switching the constraints of the $i$-th open-loop optimization problem for active FI, the nominal state $\bar{x}_{k_{d}}^{i}$ should be considered for the sake of feasibility.

Proposition 3. (Transient-state feasibility) During FI, if $\bar{x}_{k}^{i} \in \bar{X}_{f_{T}}^{i}$ holds at time instant $k$, 34 will be always feasible at the next time instants.

Proof. Since $\bar{X}_{f_{T}}^{i}$ is a CI set of the $i$-th nominal system under the constraint sets $\bar{U}_{f}^{i}$ and $\bar{X}_{f}^{i}$ and the $i$-th optimisation problem is open-loop, $\bar{x}_{k}^{i} \in \bar{X}_{f_{T}}^{i}$ implies the feasibility of the optimization problem at all the next time instants according to the definition of the CI sets.

For the proposed FI strategy, the constraint sets of $i$-th open-loop optimization should be adjusted for FI implementation at the FD time $k_{d}$. Thus, based on Proposition 3, the following strategy is proposed to guarantee the feasibility of the MPC controller during FI:

- If $\bar{x}_{k_{d}}^{i} \in \bar{X}_{f_{T}}^{i}$, (34) is always feasible during the FI process according to Proposition 3 
- If $\bar{x}_{k_{d}}^{i} \notin \bar{X}_{f_{T}}^{i}$, the center of $\bar{X}_{f_{T}}^{i}$ is used to update (34) to guarantee feasibility at time instant $k_{d}$. For $k>k_{d}$, at one time instant $k *$, if $\bar{x}_{k^{*}}^{i} \in \bar{X}_{f}^{i}$, the feasibility of (34) can always be guaranteed for all $k>k^{*}$. Otherwise, the center of $\bar{X}_{f_{T}}^{i}$ is still used to update (34) till the inclusion $\bar{x}_{k}^{i} \in \bar{X}_{f_{T}}^{i}$ is satisfied at one time instant $k>k_{d}$.

The aforementioned strategy to guarantee feasibility comes from a practical viewpoint. Moreover, since set operation and representation are based on zonotopes, $\bar{X}_{f_{T}}^{i}$ should also be a zonotope and its center can always be obtained to update the open-loop optimization problem as a remedial measure.

During the second phase of the transition, the feasibility of both the open-loop optimization and constraint satisfaction problems can be guaranteed by using the aforementioned method. However, another important aspect of the proposed FTC scheme is the stability of the closed-loop scheme. Generally, to guarantee this feature, two points should be considered: the stability of the closed-loop dynamics, which can be guaranteed by Remark 3, and the feasibility and stability of the open-loop optimization problem (34), which can be guaranteed by using the terminal-state constraint and selecting suitable control parameters as shown in (34). Particularly, this paper follows the procedure presented by Mayne et al. (2006) in order to design a stabilizing tube-based MPC controller.

4.4. Transient-state behavior after FI. In the FTC scheme, when a fault is isolated at time instant $k_{i}$, at the same time the system should be reconfigured with a different tube-based MPC controller that corresponds to this new mode. After the controller adjusting, the FTC scheme will face the same feasibility problem as during the second phase of the transition: it is assumed that the $l$-th actuator mode is isolated. Thus, the $l$-th tube-based MPC controller with the corresponding input and state constraints should be used, the $l$-th observer is employed to obtain the state estimation, and the $l$-th nominal system is used to generate the nominal states for the $l$-th open-loop optimization problem.

In order to guarantee feasibility after system reconfiguration, two methods are proposed. The first one is similar to the second-phase transition, which uses the center of $\bar{X}_{T}^{l}$ to update the $l$-th open-loop optimization problem at one time instant when

$$
\bar{x}_{k}^{l} \in \bar{X}_{T}^{l} .
$$

The second method is to use a state value $\bar{x}_{k_{i}}^{l} \in$ $\bar{X}_{T}^{l}$ to initialize the $l$-th nominal system and open-loop optimization at the FI time instant $k_{i}$. With either of the two methods, according to Proposition 3 the feasibility of the $l$-th open-loop optimization problem can always be preserved after reconfiguration. Additionally, the system can also keep being stable during this phase.

During this third-phase transition, except for the feasibility, stability and constraint satisfaction, a right restart of the FD mechanism still needs to be guaranteed. After system reconfiguration, the closed-loop system is operating in the $l$-th mode. Thus, the restarting of the FD mechanism should be considered to monitor the mode-switching behavior in this new mode. However, since in the FTC scheme the implementation of FD is based on invariant sets, if the FD mechanism is simultaneously restarted when the system is reconfigured, it is possible that the FD strategy creates false FD alarms. This situation will appear if the signals $\tilde{y}_{k}^{l, l, l}$ and $e_{k}^{l, l, l}$ do not enter into their respective sets $\tilde{Y}^{l, l, l}$ and $E^{l, l, l}$. This implies that, for the sake of right restarting, it should be guaranteed that all signals $\tilde{y}_{k}^{l, l, l}$ and $e_{k}^{l, l, l}$ have already entered into their respective sets.

In this paper, there are also two methods to avoid false FD alarms. The first one is to set a waiting time; as long as this waiting time is sufficiently long, after the waiting time, the signals can enter into their sets and the restarting of the FD mechanism can be done in the right way. In the second one after reconfiguration,

$$
\tilde{y}_{k}^{l, l, l} \in \tilde{Y}^{l, l, l} \text { and } e_{k}^{l, l, l} \in E^{l, l, l}
$$

are tested until at a time instant both inclusions hold. Then, at this time instant, the FD mechanism is restarted in the new operating mode to avoid false FD alarms.

Remark 7. (Waiting time) The waiting time can be arbitrarily defined as long as it can assure right restarting of the FD mechanism such that the aforementioned false FD alarms can be avoided. However, it is better to define the waiting time with a proper length based on the settling time of the system.

4.5. Fault-tolerant control procedure. In previous sections, the FDI and FTC approaches have been introduced in detail. In this subsection, the key point is to make a brief summary for the proposed FTC scheme, which is presented as follows:

- It is assumed that the system is in a steady state of the $i$-th mode. The FD task consists in real-time testing whether or not (17) or (18) is violated. If no violation is detected, it is considered that the system is still in the $i$-th mode. Otherwise, it is implied that a fault has occurred in the system.

- Once a fault is detected at time instant $k_{d}$, the active FI approach will be started up to isolate the fault by adjusting the constraints of (34) from $\bar{X}^{i}, \bar{U}^{i}$ and $\bar{X}_{T}^{i}$ to $\bar{X}_{f}^{i}, \bar{U}_{f}^{i}$ and $\bar{X}_{f T}^{i}$, respectively, to satisfy the 


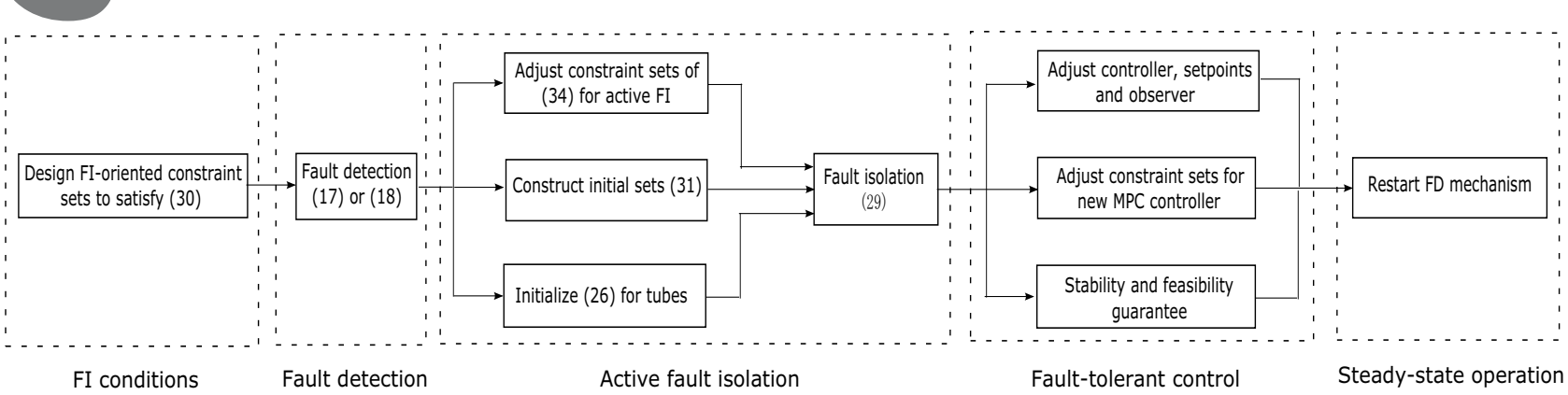

Fig. 2. Flow chart of FTC.

FI conditions on-line. Notice that the corresponding methods to guarantee the feasibility should be used during this phase.

- Simultaneously, at $k=k_{d}, p$ output-estimation-error tubes (26) are initialized by using initial sets constructed by (31). For each tube, 29) is tested in real time. Whenever a tube violates (29), the index of this tube is removed from the fault candidates until there is one and only one tube left, which implies that the fault is isolated and that the index of this tube indicates the fault.

- Once the fault is isolated (it is assumed that the fault is indexed by $l$ ), the $l$-th observer, the $l$-th tube-based MPC controller and the $l$-th state-input pair are selected to reconfigure the system (now the constraint sets should be $\bar{X}_{f}^{l}, \bar{U}_{f}^{l}$ and $\bar{X}_{f_{T}}^{l}$ for the new MPC controller, respectively). Notice that the corresponding methods to guarantee the feasibility of the $l$-th open-loop optimization and the right restarting of the FD mechanism should be used.

- After the system enters the steady state of the $l$-th mode, the whole working procedure of the proposed scheme will be revisited to monitor this new mode and the control objective is to regulate the system around the corresponding setpoint.

To further help the readers understand the the approach proposed in this paper, a flow chart describing the FTC procedure is presented in Fig. 2, where the whole procedure is divided into five steps: FI conditions, fault detection, active fault isolation, fault-tolerant control and steady-state operation.

\section{Illustrative example}

A two-tank system taken from the work of Steffen (2005), shown in Fig. 3, is used as an example to illustrate the proposed FTC scheme. The mathematical model of this two-tank system can be found in the work of Osella et al.

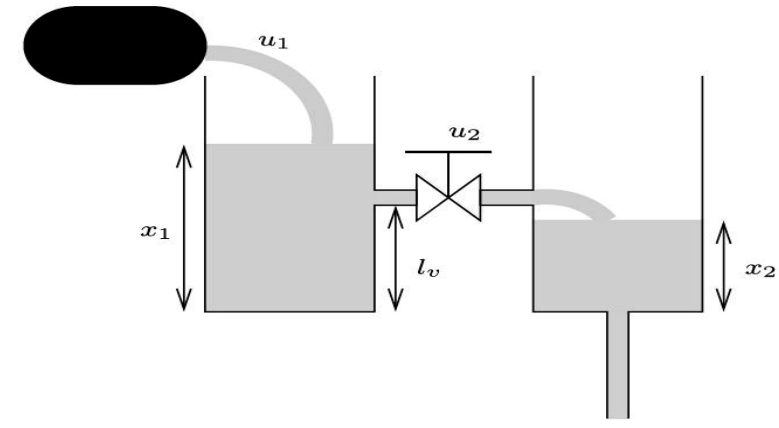

Fig. 3. Two-tank system.

(2015). With a sampling time of $0.01 \mathrm{~s}$, the dynamics of the system can be represented in discrete-time form as

$$
\begin{aligned}
x_{k+1} & =A_{d} x_{k}+B_{d} F_{i} u_{k}+E_{d} \omega_{k}, \\
y_{k} & =C_{d} x_{k}+\eta_{k},
\end{aligned}
$$

with

$$
\begin{aligned}
A_{d} & =\left[\begin{array}{cc}
0.975 & 0 \\
0.025 & 0.975
\end{array}\right], & C_{d} & =\left[\begin{array}{ll}
1 & 0 \\
0 & 1
\end{array}\right] \\
B_{d} & =\left[\begin{array}{cc}
0.1 & -0.05 \\
0 & 0.05
\end{array}\right], & E_{d} & =\left[\begin{array}{cc}
0.1 & 0 \\
0 & 0.1
\end{array}\right]
\end{aligned}
$$

where $F_{i}$ is used to model the actuator status (healthy or faulty), and it is further assumed that $|\omega| \leq$ $\left[\begin{array}{ll}0.001 & 0.001\end{array}\right]^{T}$ and $|\eta| \leq\left[\begin{array}{ll}0.001 & 0.001\end{array}\right]^{T}$.

In this case study, faults in actuators are considered. In total, there are three actuator modes taken into account, i.e., $F_{0}$ (healthy mode), $F_{1}$ (a fault in the first actuator), and $F_{2}$ (a fault in the second actuator):

$$
F_{0}=\left[\begin{array}{ll}
1 & 0 \\
0 & 1
\end{array}\right], \quad F_{1}=\left[\begin{array}{cc}
0.5 & 0 \\
0 & 1
\end{array}\right], \quad F_{2}=\left[\begin{array}{cc}
1 & 0 \\
0 & 0.5
\end{array}\right] .
$$

The water levels of the two tanks should vary within a range because of the physical limitations. Moreover, actuators also have a limited range of operation. Thus, the 


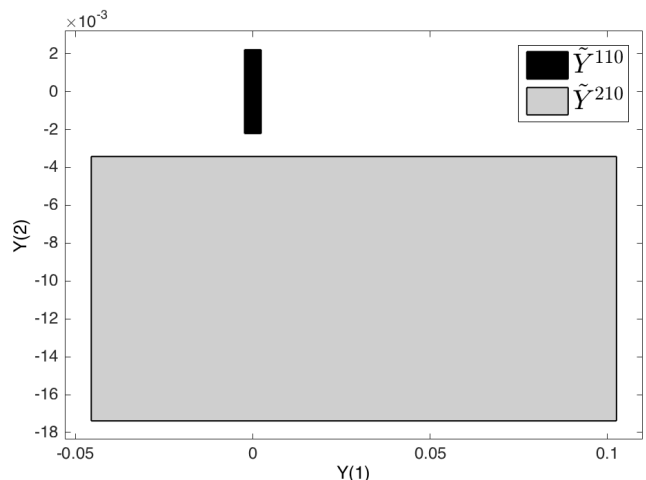

(a)

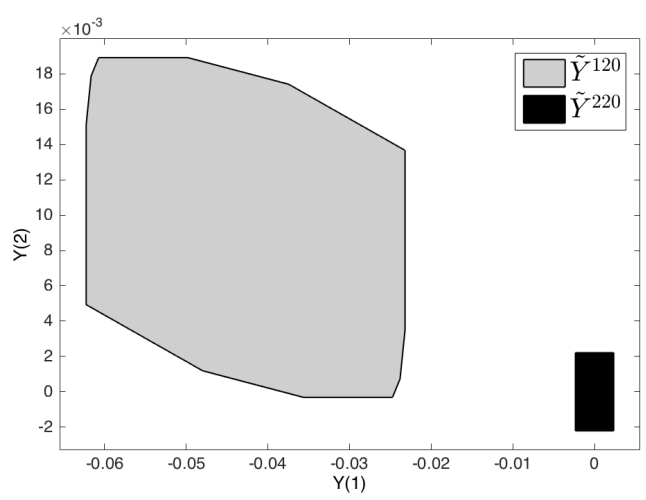

(b)

Fig. 4. After-fault sets with active FI.

system state and input constraints describing the limits of water levels and valves are set as

$$
\begin{aligned}
& U=\left\{u:\left[\begin{array}{l}
-1 \\
-1
\end{array}\right] \leq u \leq\left[\begin{array}{l}
1 \\
1
\end{array}\right]\right\}, \\
& X=\left\{x:\left[\begin{array}{l}
-1 \\
-1
\end{array}\right] \leq x \leq\left[\begin{array}{l}
1 \\
1
\end{array}\right]\right\} .
\end{aligned}
$$

Based on (37), three observers with the form 8) are designed, each matching one actuator mode. With no loss of generality, the same poles are defined for the three observers for simplicity, i.e., $p=[0.2,0.1]^{T}$. Thus, the three designed observer gains are

$$
L_{0}=L_{1}=L_{2}=\left[\begin{array}{cc}
0.775 & 0 \\
0.025 & 0.875
\end{array}\right] \text {. }
$$

Accordingly, three tube-based MPC controllers corresponding to the three modes are designed to control the system, whose feedback gains are designed as

$$
\begin{aligned}
& K_{0}=\left[\begin{array}{cc}
-0.7913 & -0.3189 \\
0.2199 & -0.4766
\end{array}\right], \\
& K_{1}=\left[\begin{array}{cc}
-0.6727 & -0.3012 \\
0.3532 & -0.4109
\end{array}\right], \\
& K_{2}=\left[\begin{array}{cc}
-0.8097 & -0.3052 \\
0.1161 & -0.3142
\end{array}\right] .
\end{aligned}
$$

In this example, the output setpoints for the three actuator modes are given as

$$
y_{0}^{*}=y_{1}^{*}=y_{2}^{*}=\left[\begin{array}{c}
0.1 \\
0.05
\end{array}\right] \text {. }
$$

Associated with these output setpoints, the state and input setpoint pairs are

$$
\begin{array}{ll}
x_{0}^{*}=\left[\begin{array}{c}
0.1 \\
0.05
\end{array}\right], & u_{0}^{*}=\left[\begin{array}{c}
0.0125 \\
-0.025
\end{array}\right], \\
x_{1}^{*}=\left[\begin{array}{c}
0.1 \\
0.05
\end{array}\right], & u_{1}^{*}=\left[\begin{array}{c}
0.025 \\
-0.025
\end{array}\right], \\
x_{2}^{*}=\left[\begin{array}{c}
0.1 \\
0.05
\end{array}\right], & u_{2}^{*}=\left[\begin{array}{l}
0.0125 \\
-0.05
\end{array}\right] .
\end{array}
$$

In this example, two fault scenarios are considered, each one corresponding to one actuator fault:

- Scenario 1: from time instants 1 to 75 , the system is healthy, and from 76 to 150 , the first actuator fault occurs.

- Scenario 2: from time instants 1 to 75 , the system is healthy, and from 76 to 150 , the second actuator fault occurs.

For these two scenarios, after a fault occurrence, a pair of active FI input and state sets need to be designed

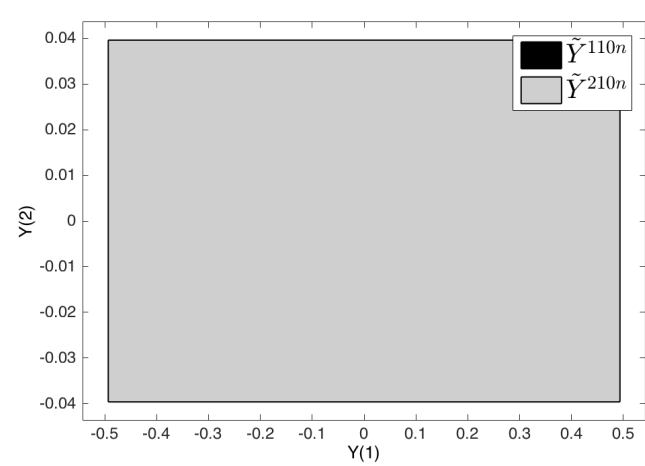

(a)

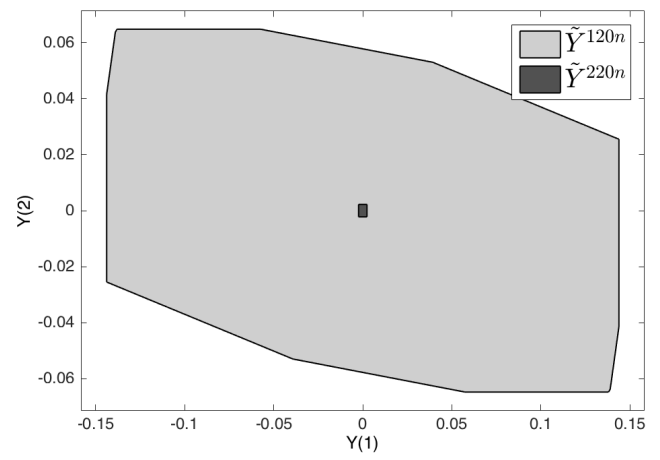

(b)

Fig. 5. After-fault sets without active FI. 
for the nominal MPC optimization problem of the healthy tube-based MPC controller, which are presented as

$$
\begin{aligned}
& \bar{U}_{f}^{0}=\left\{u:\left[\begin{array}{l}
0.2 \\
0.2
\end{array}\right] \leq u \leq\left[\begin{array}{l}
0.4 \\
0.4
\end{array}\right]\right\} \\
& \bar{X}_{f}^{0}=\left\{x:\left[\begin{array}{c}
-0.973 \\
-0.9306
\end{array}\right] \leq x \leq\left[\begin{array}{c}
0.973 \\
0.9306
\end{array}\right]\right\} .
\end{aligned}
$$

Remark 8. (State-input sets for active FI) The set pair $\bar{U}_{f}^{0}$ and $\bar{X}_{f}^{0}$ is not unique. If only the implementation of FI is considered, any set pair that satisfies the proposed FI conditions can be used for active FI.

With no loss of generality, only scenarios from healthy to faulty are considered to illustrate this FTC scheme. Thus, corresponding to $\bar{U}_{f}^{0}$, the after-fault sets of output-estimation errors of the two actuator-fault modes switched from the healthy mode can be constructed, which are shown in Fig. 4. The figure shows that the active FI set $\bar{U}_{f}^{0}$ can satisfy the guaranteed FI conditions in Proposition 2 This implies that, after detection of either of the two faults, it is guaranteed that the fault can be isolated by using the proposed FI approach.

For comparison, Fig. 5 demonstrates the after-fault sets without active FI. In this case, the after-fault output-estimation-error sets from the healthy mode should be constructed by using the input set $\bar{U}^{0}$ that can be computed by (32). In Fig. 5, the sets $\tilde{Y}^{110 n}$ and $\tilde{Y}^{220 n}$ are relatively small, and this shows that if the proposed active FI strategy is not used, it cannot be guaranteed to isolate the faults after FD.

Note that, in Figs. 4 and 5 the sets $\tilde{Y}^{110}, \tilde{Y}^{210}, \tilde{Y}^{220}$, $\tilde{Y}^{110 n}, \tilde{Y}^{210 n}$ and $\tilde{Y}^{220 n}$ are outer-bounding interval hulls of the corresponding invariant sets for simplicity of computation, which do not affect the checking of the proposed FI conditions.

Remark 9. (Notation) In Figs. 6 11, we employ symbols $E^{000}(l), \quad e_{k}^{i 00}(l), \quad \tilde{Y}^{000}(l), \quad \tilde{y}_{k}^{i 00}(l), \quad \tilde{Y}_{k}^{111}(l)$, $\tilde{Y}_{k}^{222}(l), \tilde{y}_{k}^{i 10}(l), \tilde{y}_{k}^{i 20}(l)$ and $y(l), u(l)$ to denote the $l$-th components of $E^{0,0,0}, e_{k}^{i, 0,0}, \tilde{Y}^{0,0,0}, \tilde{y}_{k}^{i, 0,0}, \tilde{Y}_{k}^{1,1,1}, \tilde{Y}_{k}^{2,2,2}$, $\tilde{y}_{k}^{i, 1,0}, \tilde{y}_{k}^{i, 2,0}, y$ and $u$, respectively. Since the output matrix is the identity matrix, the figure of system states is omitted here for simplicity.

For the first fault scenario, the FD results are shown in Fig. 6, where $\tilde{y}_{86}^{i 00}(1) \notin \tilde{Y}^{000}(1)$ indicates that a fault is detected at this time instant. Thus, the proposed active FI process is activated at the time instant $k=86$. Furthermore, it is obtained that $\tilde{y}_{87}^{i 10} \in \tilde{Y}_{87}^{111}$ and $\tilde{y}_{87}^{i 20} \notin$ $\tilde{Y}_{87}^{222}$ hold, which implies that the fault in the first actuator has occurred. Then the whole system is reconfigured to tolerate the fault. Accordingly, the inputs and outputs of Scenario 1 are presented in Fig. 8, which shows that the proposed FTC scheme can tolerate this fault with

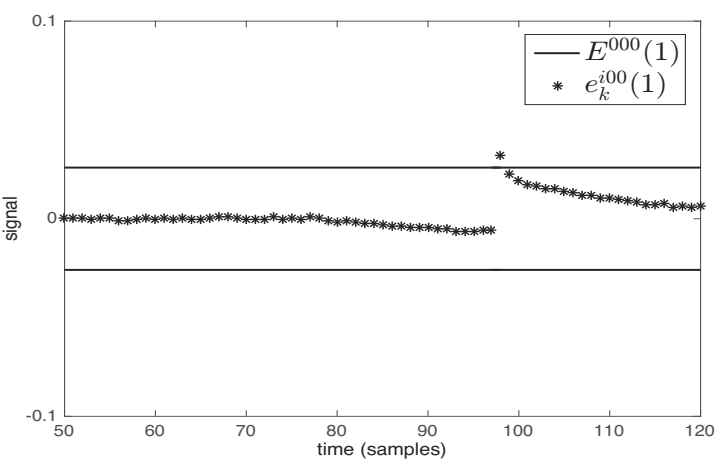

(a)

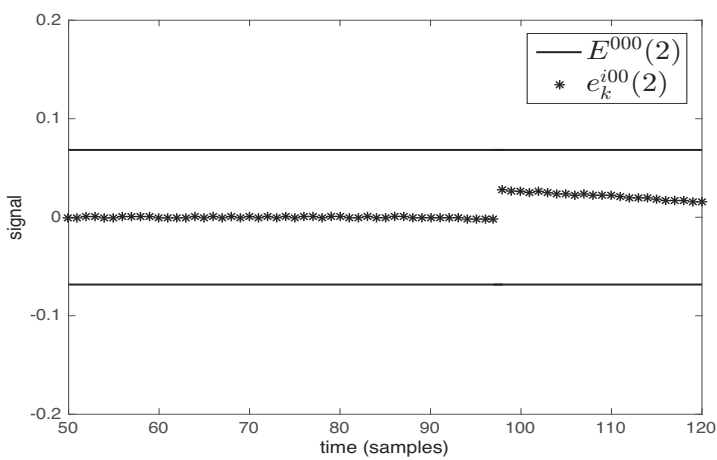

(b)

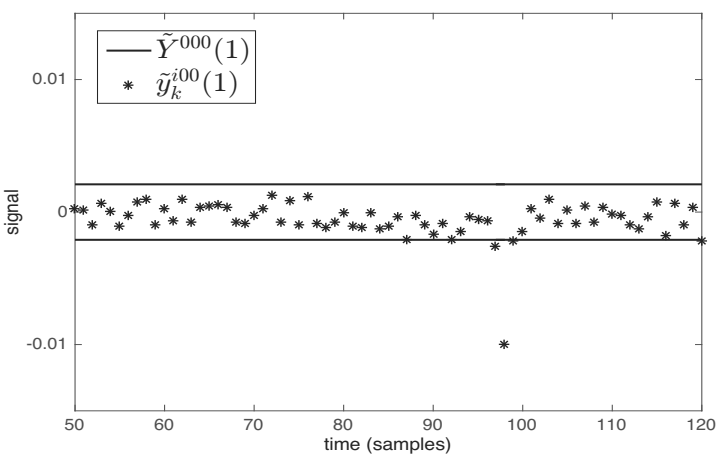

(c)

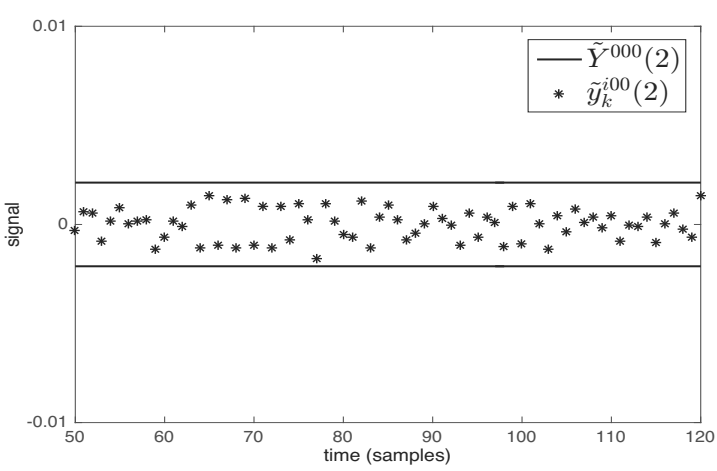

(d)

Fig. 6. FD of Fault 1. 


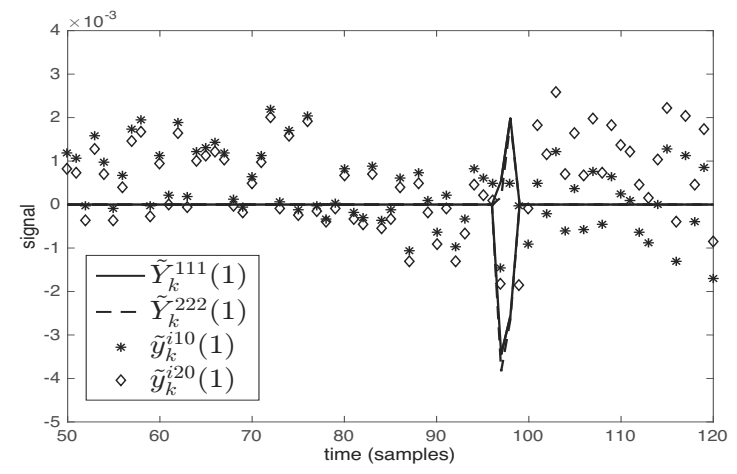

(a)

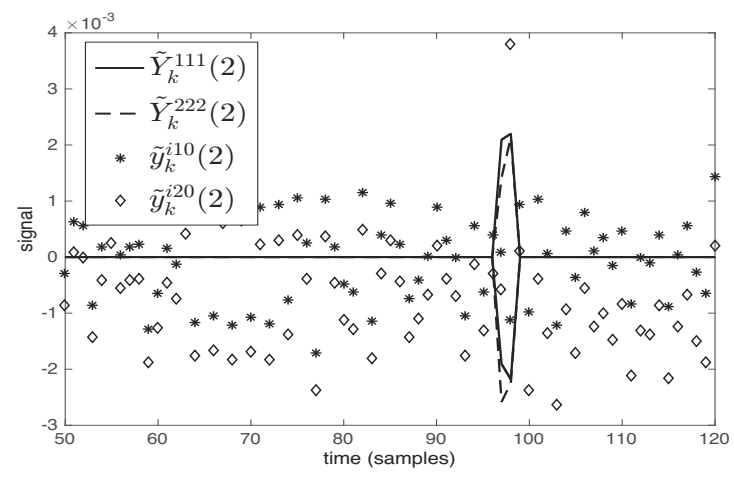

(b)

Fig. 7. FI of Fault 1.

satisfactory performance. Moreover, all the constraints can be well satisfied during the whole process.

For the second scenario, the simulation results are shown in Figs. 9-11. In Fig. 9, it is shown that an actuator fault is detected at time instant $k=80$ because $\tilde{y}_{80}^{i 00}(1) \notin$ $\tilde{Y}^{000}(1)$ is detected. Thus, at the FD time $k=80$, the active FI process is started as seen in Fig. 10. Similarly, in Fig. 10, $\tilde{y}_{k}^{i 10}$ and $\tilde{y}_{k}^{i 20}$ correspond to the first and second observers, respectively. It can be observed that $\tilde{y}_{81}^{i 10} \notin$ $\tilde{Y}_{81}^{111}$ and $\tilde{y}_{81}^{i 20} \in \tilde{Y}_{81}^{222}$ hold, which implies that the second actuator fault is isolated at time instant $k=81$. Once the second fault is isolated, the system is reconfigured by the corresponding MPC controller and state-input setpoint pair. The results in Fig. 11 show that, although the output performance has a slight degradation, the AFTC strategy can generally obtain satisfactory performance and the constraints are always well satisfied.

Remark 10. (Restarting of the FD mechanism) In order to avoid false fault alarms, whenever the system is reconfigured, a waiting time of 20 sampling times is set. During the waiting time, the FD mechanism is frozen till this period elapses. Then the FD mechanism is restarted again to monitor a new mode.

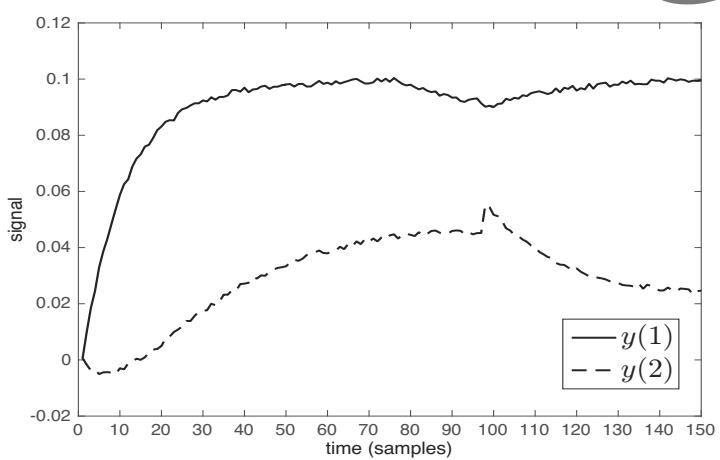

(a)

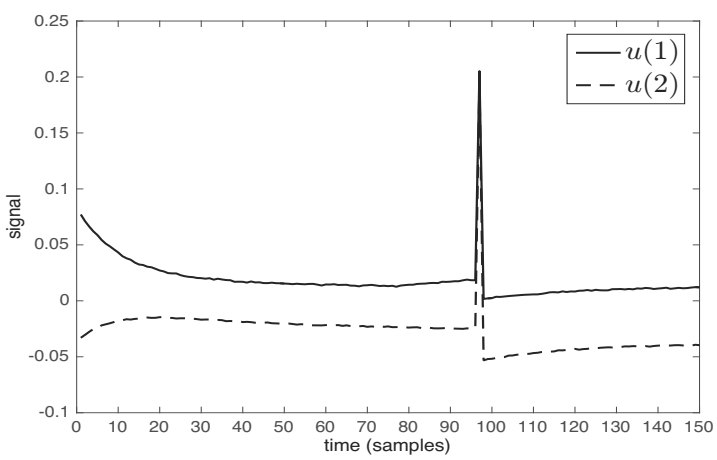

(b)

Fig. 8. System outputs and inputs of Scenario 1.

\section{Conclusions}

In this paper, an actuator FTC scheme combining tube-based MPC and set-theoretic FDI was proposed. In the scheme, FD is passive by using invariant sets and FI is active by relying on MPC and tubes, which is the most important contribution of this paper. The use of tube-based MPC and set-theoretic FDI is interesting because of their relatively low computational complexity, FDI robustness and their proper combination to implement the proposed active FI strategy. Thus, the proposed FTMPC scheme owns robust FDI performance, low computational complexity and less conservative FI conditions. The key idea of this FTC scheme consists in designing the input and state sets for active FI. In this paper, these sets are chosen by off-line trial and error as a pragmatic method, which can be improved if a systematic designing method can be proposed for the input and state FI sets in the future.

It should be emphasized that the proposed FTC scheme cannot detect all faults. Thus, for undetectable faults, the PFTC ability of this scheme can still tolerate them to some extent even though a possible degree of performance degradation may appear. Due to tube-based MPC, the advantages of the proposed FTC scheme consist in its relatively simple structure and less conservative active FI. In the future, the authors will focus on designing 


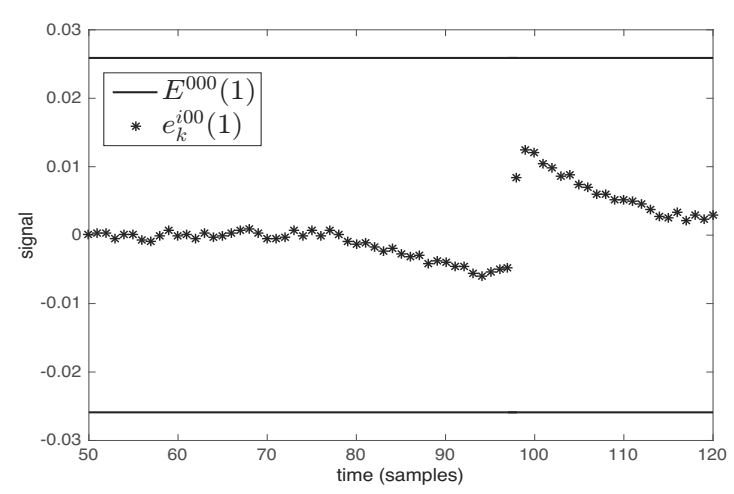

(a)

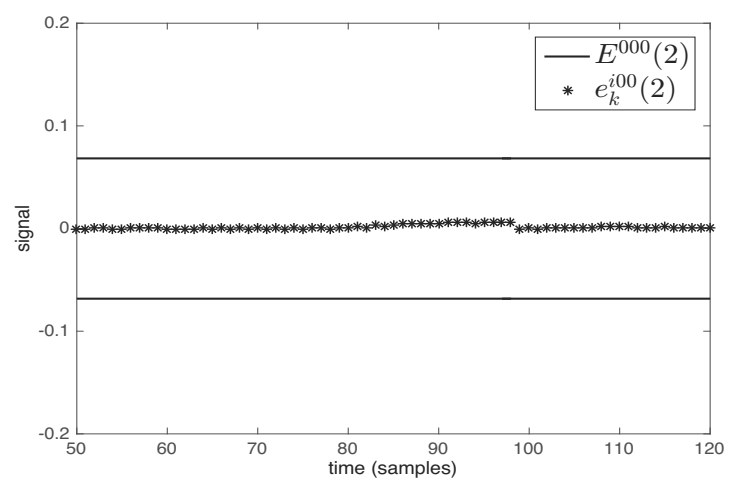

(b)

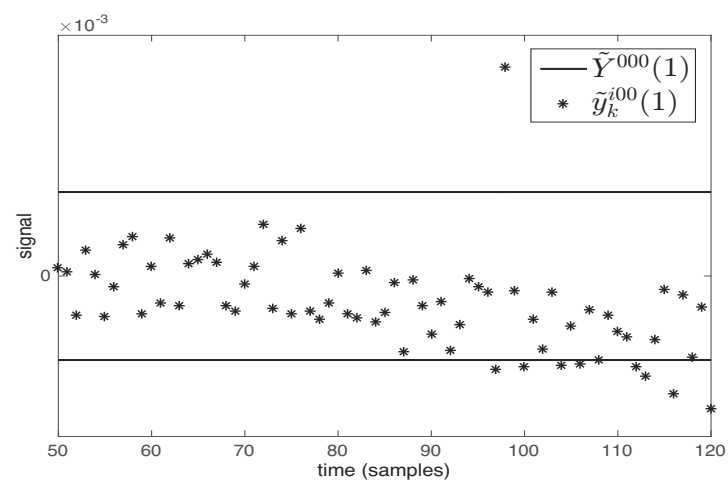

(c)

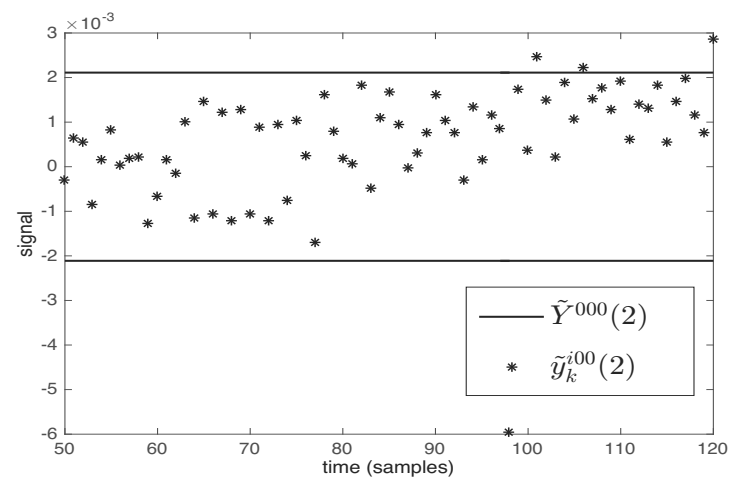

(d)

Fig. 9. FD of Fault 2.

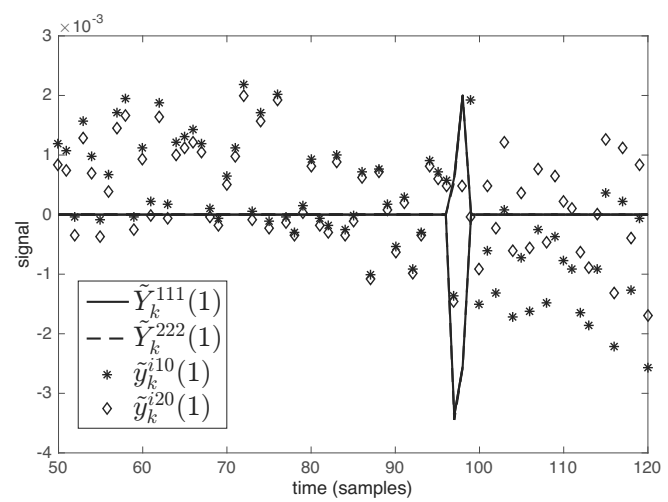

(a)

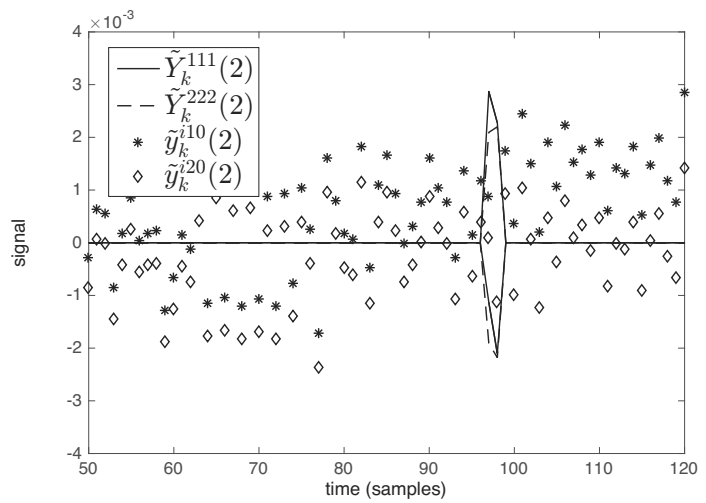

(b)

Fig. 10. FI of Fault 2.

state-input-constraint sets to further improve this FTC scheme.

\section{Acknowledgment}

This work was supported by the DGR of Generalitat de Catalunya (SAC group ref. 2014/SGR/0375), the Spanish projects ECOCIS (ref. DPI2013-48243-C2-1-R) and HARCRICS (ref. DPI2014-58104-R), the China Scholarship Council (CSC), the Automatic Control Department of Supélec and CNRS-Supélec, France.

\section{References}

Alamo, T., Bravo, J. and Camacho, E. (2005). Guaranteed state estimation by zonotopes, Automatica 41(6): 1035-1043.

Blanke, M., Kinnaert, M., Lunze, J. and Staroswiecki, M. (2006). Diagnosis and Fault-Tolerant Control, Springer-Verlag, Berlin.

Borrelli, F., Bemporad, A. and Morari, M. (2013). Predictive Control for Linear and Hybrid Systems, Model Predictive Control Lab, UC, Berkeley, CA.

Boskovic, J. and Mehra, R. (2002). Fault accommodation using model predictive methods, Proceedings of the 2002 American Control Conference, Anchorage, AK, USA, Vol. 6, pp. 5104-5109. 


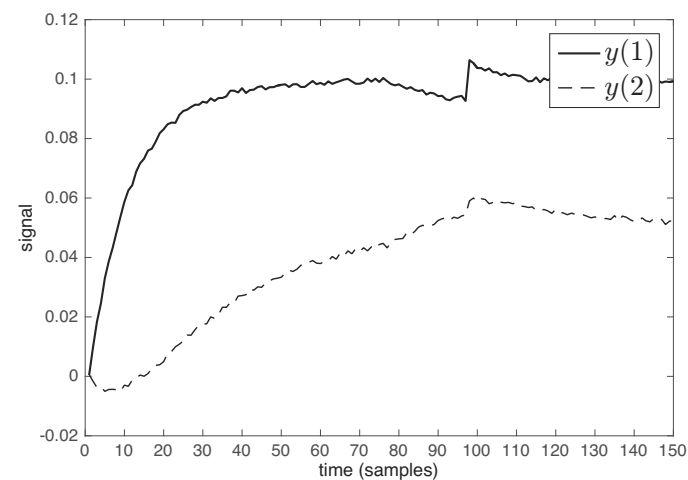

(a)

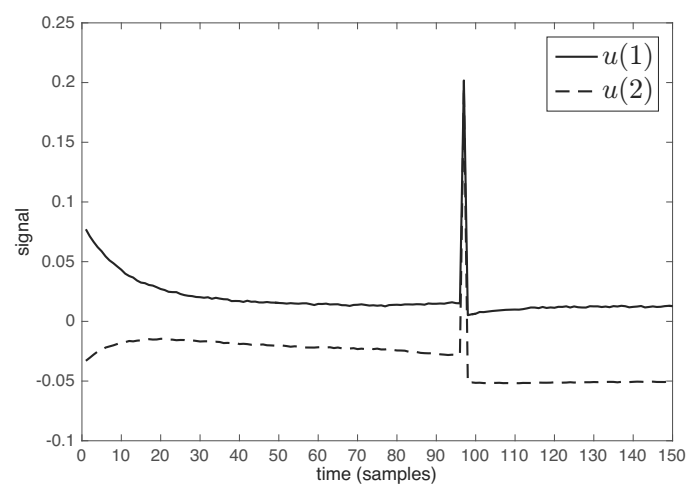

(b)

Fig. 11. System outputs and inputs of Scenario 2.

Hanlon, P. and Maybeck, P. (2000). Multiple-model adaptive estimation using a residual correlation Kalman filter bank, IEEE Transactions on Aerospace and Electronic Systems 36(2): 393-406.

Jiang, B. and Chowdhury, F. (2005). Fault estimation and accommodation for linear MIMO discrete-time systems, IEEE Transactions on Control Systems Technology 13(3): 493-499.

Jiang, B., Staroswiecki, M. and Cocquempot, V. (2006). Fault accommodation for nonlinear dynamic systems, IEEE Transactions on Automatic Control 51(9): 1578-1583.

Kofman, E., Haimovich, H. and Seron, M. (2007). A systematic method to obtain ultimate bounds for perturbed systems, International Journal of Control 80(2): 167-178.

Kolmanovsky, I. and Gilbert, E. (1998). Theory and computation of disturbance invariant sets for discrete-time linear systems, Mathematical Problems in Engineering 4(4): 317-367.

Le, V., Stoica, C., Alamo, T., Camacho, E. and Dumur, D. (2013). Zonotope-based set-membership estimation for multi-output uncertain systems, Proceedings of the 2013 IEEE international Symposium on Intelligent Control (ISIC), Hyderabad, India, pp. 212-217.

Maciejowski, J. (1999). Fault-tolerant aspects of MPC, IEE TwoDay Workshop on Model Predictive Control: Techniques and Applications, London, UK, pp. 1/1-1/4.
Mayne, D., Raković, S., Findeisen, R. and Allgöwer, F. (2006). Robust output feedback model predictive control of constrained linear systems, Automatica 42(7): 1217-1222.

Ocampo-Martinez, C., Doná, J.D. and Seron, M. (2010). Actuator fault-tolerant control based on set separation, International Journal of Adaptive Control and Signal Processing 24(12): 1070-1090.

Olaru, S., Doná, J.D., Seron, M. and Stoican, F. (2010). Positive invariant sets for fault tolerant multisensor control schemes, International Journal of Control 83(12): 2622-2640.

Osella, E., Haimovich, H. and Seron, M. (2015). Integration of invariant-set-based FDI with varying sampling rate virtual actuator and controller, International Journal of Adaptive Control and Signal Processing 30(2): 393-411.

Raimondo, D., Marseglia, G., Braatz, R. and Scott, J. (2013). Fault-tolerant model predictive control with active fault isolation, Proceedings of the 2013 Conference on Control and Fault-Tolerant Systems (SysTol), Nice, France, pp. 6567-6572.

Reppa, V., Olaru, S. and Polycarpou, M.M. (2015). Structural detectability analysis of a distributed sensor fault diagnosis scheme for a class of nonlinear systems, IFACPapersOnLine 48(21): 1485-1490.

Steffen, T. (2005). Control Reconfiguration of Dynamical Systems, Springer, Berlin.

Sun, S., Dong, L., Li, L. and Gu, S. (2008). Fault-tolerant control for constrained linear systems based on MPC and FDI, International Journal of Information and Systems Sciences 4(4): 512-523.

Xu, D., Jiang, B. and Shi, P. (2012). Nonlinear actuator fault estimation observer: An inverse system approach via a T-S fuzzy model, International Journal of Applied Mathematics and Computer Science 22(1): 183-196, DOI: 10.2478/v10006-012-0014-9.

Xu, F., Puig, V., Ocampo-Martinez, C., Olaru, S. and Nicolescu, S. (2014). Robust MPC for actuator-fault tolerance using set-based passive fault detection and active fault isolation, Proceedings of the IEEE Conference on Decision and Control, Los Angeles, CA, USA, pp. 4959-4964.

Yang, X. and Maciejowski, J.M. (2015). Fault tolerant control using Gaussian processes and model predictive control, International Journal of Applied Mathematics and Computer Science 25(1): 133-148, DOI: 10.1515/amcs-2015-0010.

Yetendje, A., Seron, M.M. and Doná, J.A.D. (2011). Robust MPC multicontroller design for actuator fault tolerance of constrained systems, IFAC Proceedings Volumes 44(1): $4678-4683$.

Yetendje, A., Seron, M.M. and De Doná, J.A. (2012). Robust multisensor fault tolerant model-following MPC design for constrained systems, International Journal of Applied Mathematics and Computer Science 22(1): 211-223, DOI: 10.2478/v10006-012-0016-7. 


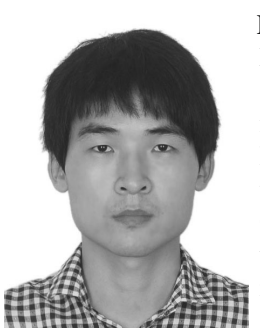

Feng Xu received his Bachelor's degree (with honors) in measurement and control technology $\&$ instruments from the Northwestern Polytechnical University (NWPU), Xi'an, China, in 2010. In 2014, he obtained his $\mathrm{PhD}$ (with honors) in automatic control from the Technical University of Catalonia (UPC), Barcelona, Spain. From January to April 2014, he was a visiting PhD student in Centrale Supélec, Paris, France. Since March 2015, he has been a postdoctoral researcher at Tsinghua University, China. His research interests include diagnosis and fault-tolerant control

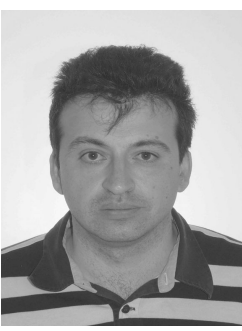

Vicenç Puig received a telecommunications engineering degree in 1993 and a $\mathrm{PhD}$ degree in automatic control, vision, and robotics in 1999, both from Universitat Politècnica de Catalunya (UPC). He is a professor at the Automatic Control Department and a researcher at Institut de Robòtica i Informàtica Industrial at UPC. He is the chair of the Automatic Control Department and the head of the research group on advanced control systems at UPC. He has developed important scientific contributions in the areas of fault diagnosis and fault tolerant control using interval and linear-parameter-varying models with set-based approaches. He has participated in more than 20 European and national research projects in the last decade. He has also led many private contracts with several companies and has published more than 100 journal articles as well as 350 papers in international conference/workshop proceedings. He has supervised over $15 \mathrm{PhD}$ dissertations and more than 40 Master's theses/final projects. He is currently the vice-chair of the IFAC Safeprocess TC Committee 6.4 (2014-2017). He is the chair of the 3rd IEEE Conference on Control and Fault-Tolerant Systems (Systol 2016) and the IPC chair of IFAC Safeprocess 2018.

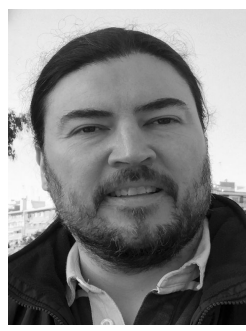

Carlos Ocampo-Martinez received an electronics engineering degree and an MSc degree in industrial automation, both from Universidad $\mathrm{Na}-$ cional de Colombia, Campus Manizales, in 2001 and 2003, respectively. In 2007 , he received a $\mathrm{PhD}$ degree in control engineering from the Technical University of Catalonia (Barcelona, Spain). Since 2011, he has been with UPC, Automatic Control Department (ESAII), as an associate professor of automatic control and model predictive control. Since 2014, he has also been a deputy director of Institut de Robòtica i Informàtica Industrial (CSIC-UPC), a Joint Research Center of UPC and CSIC. His main research interests include constrained model predictive control, large-scale systems management (partitioning and non-centralized control), and industrial applications (mainly related to the key scopes of water and energy).

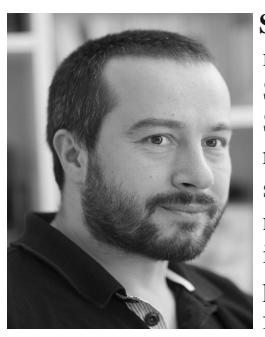

Sorin Olaru is a professor in Centrale Supélec, a member of the CNRS Laboratory of Signals and Systems and of the INRIA team DISCO (ParisSaclay University, France). His research interests include optimization-based control design, set-theoretic characterization of constrained dynamical systems as well as numerical methods in control. He is currently involved in research projects related to embedded predictive control, fault tolerant control and time-delay systems.

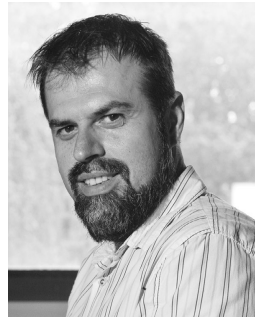

Silviu-Iulian Niculescu received a BSc degree from the Polytechnical Institute of Bucharest, Romania, and MSc and $\mathrm{PhD}$ degrees, in automatic control, from INP Grenoble, France, in 1992, 1993 and 1996, respectively. From 1992 to 1997 , he was with the University "Politehnic" Bucharest. From 1997 to 2006, he was with the HEUDIASYC (heuristics and diagnosis of complex systems) laboratory, Compiègne, France, as a researcher at CNRS (French National Cente for Scientific Research). In September 2006, he joined L2S (Laboratory of Signals and Systems), Gif-sur-Yvette, where he is currently a research director at CNRS and the head of the laboratory. His research interests include delay systems, robust control, and numerical methods in optimization and their applications to the design of engineering systems.

\section{Appendix}

Definition A1. (Zonotopes) An $r$-order zonotope $Z$ is defined as $Z=g \oplus H \mathbb{B}^{r}$, where $g$ and $H$ are its center and segment matrix (or generator matrix), respectively.

Definition A2. (Interval hull) The interval hull $\square Z$ of a zonotope $Z=g \oplus H \mathbb{B}^{r} \subset \mathbb{R}^{n}$ is the smallest box containing $Z$, i.e.,

$$
\square Z=\left\{x:\left|x_{i}-g_{i}\right| \leq\left\|H_{i}\right\|_{1}\right\},
$$

where $H_{i}$ is the $i$-th row of $H, x_{i}$ and $g_{i}$ are the $i$-th components of $x$ and $g$, respectively.

Property A1. (Minkowski sum of zonotopes) Given two zonotopes $Z_{1}=g_{1} \oplus H_{1} \mathbb{B}^{r_{1}} \subset \mathbb{R}^{n}$ and $Z_{2}=g_{2} \oplus$ $H_{2} \mathbb{B}^{r_{2}} \subset \mathbb{R}^{n}, Z_{1} \oplus Z_{2}=\left(g_{1}+g_{2}\right) \oplus\left[\begin{array}{ll}H_{1} & H_{2}\end{array}\right] \mathbb{B}^{r_{1}+r_{2}}$.

Property A2. (Multiplication of zonotopes) Given a zonotope $Z=g \oplus H \mathbb{B}^{r} \subset \mathbb{R}^{n}$ and a suitable matrix $K$, $K Z=K g \oplus K H \mathbb{B}^{r}$.

Property A3. (Reordering of zonotopes) Given a zonotope $Z=g \oplus H \mathbb{B}^{r} \subset \mathbb{R}^{n}$ and an integer $s$ (with $n<s<r$ ), denote by $\hat{H}$ the matrix resulting from the reordering of the columns of the matrix $H$ in decreasing Euclidean norm. $Z \subseteq g \oplus\left[\hat{H}_{T} \quad Q\right] \mathbb{B}^{s}$ where $\hat{H}_{T}$ is obtained from the first $s-n$ columns of the matrix $\hat{H}$ and $Q \in \mathbb{R}^{n \times n}$ is a diagonal matrix whose elements satisfy $Q_{i i}=\sum_{j=s-n+1}^{r}\left|\hat{H}_{i j}\right|, i=1, \ldots, n$.

Property A4. (Intersection of a zonotope and a strip) Given a zonotope $Z=g \oplus H \mathbb{B}^{r} \subset \mathbb{R}^{n}$, a strip $S=$ $\left\{x \in \mathbb{R}^{n}|| c x-d \mid \leq \sigma\right\}$ and a vector $\lambda \in \mathbb{R}^{n}$, then $Z \cap S \subseteq \hat{Z}(\lambda)=\hat{g}(\lambda) \oplus \hat{H}(\lambda) \mathbb{B}^{r+1}$ holds, where $\hat{g}(\lambda)=g+\lambda(d-c g)$ and $H(\lambda)=\left[\begin{array}{ll}(I-\lambda c) H & \sigma \lambda\end{array}\right]$.

Property A5. (Intersection of a zonotope and a polytope) Given a matrix $\Lambda \in \mathbb{R}^{n \times m}$, a zonotope $Z=g \oplus H \mathbb{B}^{r}$, and an $H$-polytope $P=\left\{x \in \mathbb{R}^{n}:|C x-d| \leq\right.$ $\left.\left[\phi_{1}, \phi_{2}, \ldots, \phi_{m}\right]^{T}\right\}$, with $C \in \mathbb{R}^{m \times n}, d \in \mathbb{R}^{m}, \phi_{i} \in \mathbb{R}_{+}$ $(i=1,2, \ldots, m)$, define a vector $\hat{g}(\Lambda)=g+\Lambda(d-C g)$ and a matrix $\hat{H}(\Lambda)=[(I-\Lambda C) H \quad \Lambda \Phi]$, with a diagonal 
matrix $\Phi=\operatorname{diag}\left(\phi_{1}, \phi_{2}, \ldots, \phi_{m}\right)$. Then a family of zonotopes (parameterized by the matrix $\Lambda$ ) that contains the intersection of the zonotope $Z$ and the polytope $P$ is obtained as $Z \cap P \subseteq \hat{Z}(\Lambda)=\hat{g} \oplus \hat{H} \mathbb{B}^{r+m}$.

Definition A3. (RPI set) A set $\mathcal{X}$ is an RPI set of the dynamics $x_{k+1}=A x_{k}+\omega_{k}$ if for $x_{k} \in \mathcal{X}$ and $\omega_{k} \in W$, $x_{k+1} \in A \mathcal{X}+W \subseteq \lambda \mathcal{X}(0<\lambda \leq 1)$ always holds.

Definition A4. (Minimal RPI set) The minimal RPI (mRPI) set of the dynamics $x_{k+1}=A x_{k}+\omega_{k}$ is defined as an RPI set contained in any closed RPI set and the mRPI set is unique and compact.

Theorem A1. (Construction of invariant sets) For the $d y$ namics $x_{k+1}=A x_{k}+B \delta_{k}$ where $A$ and $B$ are constant matrices and $A$ is a Schur matrix, $\delta_{k}$ belongs to $\Delta=\left\{\delta:\left|\delta-\delta^{\circ}\right| \leq \bar{\delta}\right\}$ with $\delta^{\circ}$ and $\bar{\delta}$ constant, letting $A=V \Lambda V^{-1}$ be the Jordan decomposition, the set

$$
\Phi(\theta)=\left\{x \in \mathbb{R}^{n}:\left|V^{-1} x\right| \leq(I-|\Lambda|)^{-1}\left|V^{-1} B\right| \bar{\delta}+\theta\right\}
$$$$
\oplus \xi^{\circ}
$$

is RPI and attractive for the state trajectories, with $\theta$ any (arbitrarily small) vector with positive components, where $\xi^{\circ}=(I-A)^{-1} B \delta^{\circ}$ :

1. For any $\theta$, the set $\Phi(\theta)$ is (positively) invariant; that is, if $x_{0} \in \Phi(\theta)$, then $x_{k} \in \Phi(\theta)$ for all $k \geq 0$.

2. Given $\theta \in \mathbb{R}^{n}, \theta>0$, and $x_{0} \in \mathbb{R}^{n}$, there exists $k^{*} \geq 0$ such that $x_{k} \in \Phi(\theta)$ for all $k \geq k^{*}$.

Definition A5. (CI set) A set $\mathcal{X} \subseteq X$ is a CI set of the dynamics $x_{k+1}=A x_{k}+B u_{k}$ if, for any $x_{k} \in \mathcal{X}$, there always exists $u_{k} \in U$ such that $x_{k+1} \in \mathcal{X}$ for all $k \geq 0$.

Definition A6. (MCI set) A set $\mathcal{X}_{\mathcal{M}} \subseteq X$ is said to be the MCI set of the dynamics $x_{k+1}=A x_{k}+B u_{k}$ if it is $\mathrm{CI}$ and contains all CI sets inside $X$.

Received: 4 May 2015

Revised: 28 December 2015

Re-revised: 27 August 2016

Accepted: 20 September 2016 